\title{
An essential role for a discrete parasubthalamic nucleus subpopulation in appetite suppression
}

\author{
Jessica H. Kim ${ }^{*}$, Grace H. Kromm ${ }^{*}$, Olivia K. Barnhill ${ }^{1}$, Kenneth Han ${ }^{1}$, Lauren B. \\ Heuer ${ }^{1}$, Sierra Loomis', Matthew C. Newman', Jacob Sperber ${ }^{1}$, Theresa B. Legan ${ }^{1}$, Faris \\ F. Gulamali ${ }^{1}$, Katharine E. Jensen ${ }^{2}$, Samuel C. Funderburk ${ }^{3}$, Michael J. Krashes ${ }^{3}$, and \\ Matthew E. Carter ${ }^{1}$
}

${ }^{1}$ Department of Biology

${ }^{2}$ Department of Physics

Williams College

Williamstown, MA, 01267 USA

${ }^{3}$ Diabetes, Endocrinology, and Obesity Branch

National Institutes of Diabetes and Digestive and Kidney Diseases

National Institutes of Health

Bethesda, MD 20892 USA

*Authors contributed equally to this work

Correspondence: Matthew E. Carter, Ph.D.

Department of Biology

Thompson Biology Lab Rm 218

Williams College

Williamstown, MA United States 01267

Email:mc10@williams.edu 


\section{Abstract}

Food intake behavior is regulated by a network of appetite-inducing and appetite-suppressing neuronal populations throughout the brain. The parasubthalamic nucleus (PSTN), a relatively unexplored population of neurons in the posterior hypothalamus, has been hypothesized to regulate appetite due to its connectivity with other anorexigenic neuronal populations and because these neurons express Fos, a marker of neuronal activation, following a meal. However, the individual cell types that make up the PSTN are not well characterized, nor are their functional roles in food intake behavior. Here we identify and distinguish between two discrete PSTN subpopulations, those that express tachykinin-1 (PSTN ${ }^{\mathrm{Tac} 1}$ neurons) and those that express corticotropin-releasing hormone (PSTN ${ }^{\mathrm{CRH}}$ neurons), and use a panel of genetically encoded tools in mice to show that PSTN ${ }^{\mathrm{Tac} 1}$ neurons play an essential role in appetite suppression. Both subpopulations increase activity following a meal and in response to administration of the anorexigenic hormones amylin, cholecystokinin (CCK), and peptide YY (PYY). Interestingly, chemogenetic inhibition of PSTN ${ }^{\mathrm{Tac} 1}$, but not PSTN ${ }^{\mathrm{CRH}}$ neurons, reduces the appetitesuppressing effects of these hormones. Consistently, optogenetic and chemogenetic stimulation of PSTN ${ }^{\mathrm{Tac} 1}$ neurons, but not PSTN ${ }^{\mathrm{CRH}}$ neurons, is sufficient to reduce food intake in hungry mice. PSTN ${ }^{\mathrm{Tac} 1}$ and PSTN ${ }^{\mathrm{CRH}}$ neurons project to distinct downstream brain regions, and stimulation of $\mathrm{PSTN}^{\mathrm{Tac} 1}$ projections to individual anorexigenic populations reduces food consumption. Taken together, these results reveal the functional properties and projection patterns of distinct PSTN cell types and demonstrate an essential, anorexigenic role for PSTN ${ }^{\text {Tac1 }}$ neurons in the hormonal and central regulation of appetite. 


\section{Introduction}

The brain regulates food intake behavior through the coordinated activity of several distinct neuronal populations (Andermann and Lowell, 2017; Sternson and Eiselt, 2017). Activity in orexigenic (appetite-inducing) populations, such as agouti-related peptide (AgRP)-expressing neurons in the hypothalamic arcuate nucleus, is necessary and sufficient to promote feeding behavior (Aponte et al., 2011; Krashes et al., 2011). In contrast, activity in anorexigenic (appetite-suppressing) populations, such as pro-opiomelanocortin (POMC)-expressing neurons in the hypothalamus (Aponte et al., 2011) or calcitonin gene related peptide (CGRP)-expressing neurons in the brainstem parabrachial nucleus (PBN ${ }^{\text {CGRP }}$ neurons) (Campos et al., 2016; Carter et al., 2013), is necessary and sufficient to suppress feeding. In recent years, the parasubthalamic nucleus (PSTN), a relatively understudied population of neurons in the posterolateral hypothalamus, has been hypothesized to regulate feeding behavior; however, the gene expression patterns within the PSTN and the role of individual cell types in food intake behavior has remained relatively uncharacterized.

The few studies that have explored the PSTN demonstrate a potential role for these neurons in feeding. Anatomically, PSTN neurons receive afferent projections from orexigenic AgRP neurons (Livneh et al., 2017; Wang et al., 2015) and anorexigenic populations including arcuate POMC neurons (Wang et al., 2015), PBN ${ }^{\text {CGRP }}$ neurons (Huang et al., 2021), and the central nucleus of the amygdala (CeA) (Barbier et al., 2017). In turn, these neurons project to anorexigenic brain regions including the PBN (Goto and Swanson, 2004; Zseli et al., 2016), CeA (Barbier et al., 2020; Zseli et al., 2017), nucleus of the solitary tract (NST) (Ciriello et al., 2008; Goto and Swanson, 2004; Holt et al., 2019), and paraventricular thalamic nucleus (PVT) (Zhang and van den Pol, 2017). PSTN neurons express Fos, an immediate early gene that serves as an 
indirect marker of neuronal activation, following a large meal (Barbier et al., 2020; Chometton et al., 2016; Zseli et al., 2016; Zseli et al., 2017), during a learned conditioned flavor aversion (Yasoshima et al., 2006), and in response to dietary amino acid deficiency that causes a decrease in appetite (Zhu et al., 2012). Optogenetic stimulation of glutamatergic projections from the PSTN to the PVT is sufficient to reduce food intake (Zhang and van den Pol, 2017). Finally, chemogenetic inhibition of PSTN neurons that express tachykinin-1 (Tac1) decreases taste neophobia (Barbier et al., 2020).

Taken together, these initial studies suggest a role for the PSTN in feeding behavior. However, the individual subpopulations that make up the PSTN remain relatively uncharacterized, as do their functional roles during food consumption. We therefore sought to characterize the cell types that make up the PSTN, determine their activity patterns in response to various appetitive stimuli, perturb their function in freely behaving mice, and map their connectivity with other brain regions. We identify two discrete populations of PSTN neurons, each with distinct influences on feeding behavior. 


\section{Results}

\section{Identification of distinct PSTN cell populations}

We initially identified the PSTN as a candidate anorexigenic population by investigating sources of afferent input to PBN ${ }^{\mathrm{CGRP}}$ neurons, a population that has previously been shown to suppress appetite and control meal termination (Campos et al., 2017; Campos et al., 2016; Carter et al., 2013; Essner et al., 2017). To retrogradely label neurons that project to $P B N^{C G R P}$ neurons, we used a cre-inducible modified rabies virus system in $\mathrm{Calca}^{\mathrm{Cr} /+}$ mice (Calca is the gene that encodes CGRP; Figures 1A and B). Retrograde expression was observed in populations previously shown to project to the PBN, including the bed nucleus of the stria terminalis (BNST) (Wang et al., 2019), central nucleus of the amygdala (CeA) (Cai et al., 2014), arcuate nucleus of the hypothalamus (Arc) (Carter et al., 2013; Essner et al., 2017; Wu et al., 2009), and nucleus of the solitary tract (NTS) (Roman et al., 2016) (Figures 1C-H). We also observed retrogradely labelled cells in the PSTN, just medial to the cerebral peduncle in the posterior hypothalamus (Figures 1G and 1I). Similar to previous studies (Barbier et al., 2020; Chometton et al., 2016; Zseli et al., 2016; Zseli et al., 2017), we found that refeeding following 18-h food deprivation induced substantial expression of Fos in the PSTN region (Figures 1J and K), suggesting that PSTN neurons play a role in appetite suppression. Therefore, to better characterize these Fosexpressing neurons, we analyzed the cell types that make up the PSTN and explored their roles in food intake behavior.

To identify potential genetic markers for PSTN neurons, we consulted the Allen Brain Explorer (http://musebrain-map.org) (Ng et al., 2009) and searched for genes enriched in the PSTN. Additionally, previous studies reported expression of Tacl (Barbier et al., 2020; WallenMackenzie et al., 2020) and Crh (Zhu et al., 2012) in the PSTN region. Indeed, the Allen Mouse 
Brain Atlas (Lein et al., 2007) demonstrates expression of Tacl and Crh within the PSTN region, with expression of Slc17a6 (the gene that encodes VGlut2), Calb1, Calb2, and Pvalb more diffusely located throughout the local area (Figures 2A).

To quantify the specificity and co-expression of genetic markers in the PSTN, we performed fluorescent in situ hybridization (FISH), initially focusing on Tacl and Crh due to the apparent specificity of these markers within the PSTN. As expected, Tacl-and Crh-expressing cells were located within the PSTN region (Figure 2B). Interestingly, there was nearly distinct expression of each marker in the PSTN, with $85.1 \%$ of labeled PSTN cells expressing Tac1, $14.9 \%$ of labeled PSTN cells expressing $C r h$, and only $1.9 \%$ of cells co-expressing both markers (Figure 2C). Therefore, PSTN ${ }^{\mathrm{Tac} 1}$ and PSTN ${ }^{\mathrm{CRH}}$ neurons constitute two nearly distinct cell populations in the PSTN.

To determine the co-expression of PSTN ${ }^{\mathrm{Tac} 1}$ and PSTN ${ }^{\mathrm{CRH}}$ neurons with other potential genetic markers, we performed triple-label FISH experiments. Every Tacl-and Crh-expressing cell also expressed Slc17a6, indicating that these populations are glutamatergic (Figures 2D and E). A majority of Tac1- and Crh-expressing cells co-expressed Calb1 and Calb2, although expression of these markers was diffuse and extended medially far beyond the PSTN region (Figures 2F-I). Less than 2\% of Tacl and Crh-expressing cells co-expressed Pvalb (Figures 2J and K). A Pvalb-expressing population appeared dorsomedial to the PSTN, while two other Pvalb populations appeared medially and laterally to the PSTN in the same horizontal plane. Therefore, Tac 1 and $\mathrm{CRH}$ neurons are specific and discrete glutamatergic subpopulations within the PSTN. 


\section{PSTN neurons increase activity in response to feeding and anorexigenic hormones}

To determine whether PSTN ${ }^{\mathrm{Tacl}}$ and/or PSTN ${ }^{\mathrm{CRH}}$ neurons are active in response to feeding, we first pursued a histological approach using in situ hybridization to measure co-expression of Fos following a large meal. Mice were food deprived for $18 \mathrm{~h}$; half the animals were then allowed to consume a large meal while the other half remained fasted, and all animals were perfused $45 \mathrm{~min}$ later to allow time for Fos mRNA expression dynamics. Animals that were not allowed to eat following the food deprivation period exhibited relatively low Fos expression in the PSTN (Figure 3A). In contrast, animals that were allowed to refeed showed robust co-expression of Fos in both Tacl and Crh populations (Figures 3B and C; See also Supplementary Table 1 for detailed statistical analysis). Although a greater percentage of Crh neurons co-expressed Fos, a significantly higher total number of Tacl neurons co-expressed Fos (Figure 3D). Taken together, these results demonstrate that refeeding increases activity as measured by Fos expression in both PSTN $^{\text {Tacl }}$ and PSTN ${ }^{\mathrm{CRH}}$ neurons.

To determine acute activity patterns in PSTN ${ }^{\mathrm{Tac} 1}$ or PSTN ${ }^{\mathrm{CRH}}$ neurons upon food exposure, we transduced PSTN neurons in $\mathrm{Tacl}^{\mathrm{Cre} /+}$ or $\mathrm{Crh}^{\mathrm{Cre} /+}$ mice with the GCaMP6s calcium indicator and measured real-time fluorescence intensity using fiber photometry (Figures 3E and F). Although we detected post-hoc GCaMP6s fluorescent expression in $\mathrm{Crh}^{\mathrm{Cre} /+}$ mice, we were not able to record reliable fluorescence signals from these mice following a variety of conditions, potentially due to the relatively low number of CRH neurons in the PSTN. Therefore, we focused on $\mathrm{Tacl}^{\mathrm{Cre} / \mathrm{+}}$ mice, recording from mice either food deprived for $18 \mathrm{~h}$ or fed ad libitum. Exposure to standard mouse chow or palatable peanut butter produced a significant increase in PSTN $^{\text {Tac1 }}$ activity in 18-h food deprived mice compared with exposure to a novel object or exposure to water in 16-h water deprived mice (Figures 3G-L). This increase in activity persisted 
for approximately the first $60 \mathrm{~s}$ upon exposure to food. These effects were diminished in $a d$ libitum fed mice that were less motivated to consume food. Taken together, these results suggest that PSTN ${ }^{\text {Tac1 }}$ neurons increase activity during the initial stages of food consumption in hungry mice, but do not respond to water in thirsty mice or other salient stimuli.

We next examined the effects of anorexigenic hormone administration on activity in PSTN $^{\text {Tac1 }}$ and PSTN ${ }^{\mathrm{CRH}}$ neurons. To measure neural activity using Fos expression, animals were food deprived for $18 \mathrm{~h}$ to reduce endogenous anorexigenic hormone levels, and then injected intraperitoneally with saline, amylin, cholecystokinin (CCK), or peptide YY (PYY).

Administration of these anorexigenic hormones caused an increase in Fos expression in both Tacl and Crh neurons compared with animals injected with saline (Figures 4A-E), indicating that these anorexigenic hormones cause increased activation of PSTN ${ }^{\mathrm{Tac} 1}$ and PSTN ${ }^{\mathrm{CRH}}$ neurons. To measure neural activity using fiber photometry, $\mathrm{Tac1} \mathrm{Cre}^{\mathrm{C}+}$ mice transduced with GCaMP6s were implanted with an intraperitoneal catheter (Figure 4F), allowing for real-time measurement of PSTN ${ }^{\text {Tac1 }}$ activity during hormone administration without the need to inject mice and potentially cause stress by physically handling animals. Administration of amylin, CCK, and PYY caused a significant increase in PSTN $^{\text {Tac1 }}$ activity compared with administration of saline (Figure 4G-L). Therefore, PSTN ${ }^{\text {Tac1 }}$ neurons increase activity during food consumption and in response to elevated levels of anorexigenic hormones.

\section{Inhibition of PSTN ${ }^{\text {Tac1 }}$ neurons attenuates the effects of anorexigenic hormones}

To determine whether activity in $\mathrm{PSTN}^{\mathrm{Tac} 1}$ or PSTN ${ }^{\mathrm{CRH}}$ neurons is necessary for normal food intake behavior, we bilaterally injected AAV carrying either Cre-inducible hM4Di-mCherry or mCherry transgenes into the PSTN of $\mathrm{Tacl}^{\mathrm{Cre} /+}$ and $\mathrm{Crh}^{\mathrm{Cre} /+}$ mice (Figures $5 \mathrm{~A}-\mathrm{C}$ ). 
In $\mathrm{Tacl}^{\mathrm{Cre} /+}$ mice, there was no difference in food consumption between animals transduced with hM4Di-mCherry and mCherry following intraperitoneal administration of clozapine $N$-oxide (CNO) and inert saline solution (Figure 5D). Therefore, inhibition of PSTN $^{\text {Tac1 }}$ neurons is insufficient to affect baseline food intake. Because anorexigenic hormones increased activity in PSTN ${ }^{\text {Tac1 }}$ neurons (Figure 4), we assessed whether this activity is necessary for their anorexigenic effects. As expected, administration of amylin, CCK, and PYY significantly reduced food intake relative to administration of saline (Figures 5D-G). Intriguingly, co-administration of CNO significantly attenuated these anorexigenic effects in hM4Di-transduced animals (Figures 5E-G), demonstrating the necessity of PSTN ${ }^{\text {Tac1 }}$ neuronal activity for the full anorexigenic effects of amylin, CCK, and PYY. To better understand how PSTN $^{\text {Tac1 }}$ neuron inhibition increases food intake in these conditions, we analyzed individual meal parameters in the first $3 \mathrm{~h}$ following injection of CNO. Administration of CNO did not affect meal size or meal duration, but increased meal frequency in hM4Di-mCherry-transduced mice (Figures 5H-J). This analysis suggests that inhibition of $\operatorname{PSTN}^{\mathrm{Tac} 1}$ neurons attenuates the reduction in food intake caused by anorexigenic hormones by increasing the frequency of meals. In contrast to $\mathrm{Tacl}^{\mathrm{Cre} /+}$ mice, there were no differences between hM4Di-mCherry and mCherrytransduced $\mathrm{Crh}^{\mathrm{Cre} /+}$ mice following administration of CNO with saline (Figure 5K) or anorexigenic compounds (Figures 5L-N). Therefore, activity in PSTN ${ }^{\mathrm{Tac} 1}$ neurons, but not PSTN $^{\mathrm{CRH}}$ neurons, is necessary for the suppression of meal frequency caused by amylin, CCK, and PYY. 


\section{PSTN $^{\text {Tac1 }}$ neurons are sufficient to suppress food intake}

To determine whether a gain of activity in $\mathrm{PSTN}^{\mathrm{Tac} 1}$ or PSTN ${ }^{\mathrm{CRH}}$ neurons is sufficient to suppress feeding, we unilaterally injected AAV carrying either Cre-inducible mCherry or ChR2mCherry transgenes into the PSTN of $\mathrm{Tacl}^{\mathrm{Cre} /+}$ and $\mathrm{Crh}^{\mathrm{Cre} /+}$ mice for optogenetic stimulation experiments (Figures 6A-C). Mice transduced with ChR2-mCherry in PSTN ${ }^{\mathrm{Tac} 1}$ neurons consumed significantly less food during the photostimulation period than mice transduced with mCherry (Figures 6D and E). In contrast, photostimulation of PSTN ${ }^{\mathrm{CRH}}$ neurons caused no observable effects on food intake (Figures 6F and G). To independently verify these effects and assess the impact of longer-term stimulation, we also examined the effects of chemogenetic stimulation of PSTN ${ }^{\mathrm{Tac} 1}$ or PSTN ${ }^{\mathrm{CRH}}$ neurons on food intake behavior by transducing these neurons with mCherry or hM3Dq-mCherry transgenes (Figures 6H-J). Following administration of CNO, mice transduced with hM3Dq-mCherry in $\operatorname{PSTN}^{\mathrm{Tac} 1}$ neurons consumed significantly less food over a $3 \mathrm{~h}$ period than mice transduced with mCherry (Figure 6K). There was no effect of CNO administration between mice transduced with hM3Dq-mCherry or mCherry in PSTN ${ }^{\mathrm{CRH}}$ neurons (Figure 6L). Therefore, these gain-of-function experiments suggest that optogenetic or chemogenetic stimulation of PSTN ${ }^{\mathrm{Tac} 1}$ neurons, but not PSTN ${ }^{\mathrm{CRH}}$ neurons, is sufficient to reduce food consumption.

\section{$\operatorname{PSTN}^{\text {Tac1 } 1}$ and PSTN ${ }^{\text {CRH }}$ neurons exhibit different projection patterns}

The necessity and sufficiency of PSTN ${ }^{\mathrm{Tac} 1}$ neurons, but not PSTN ${ }^{\mathrm{CRH}}$ neurons, in appetite suppression suggests that these neural populations have different projection targets throughout the brain. To identify and distinguish between downstream projections of $\operatorname{PSTN}^{\mathrm{Tac} 1}$ and PSTN $^{\mathrm{CRH}}$ neurons, we transduced each population with mCherry and surveyed the brain for 
anterograde fluorescence (Figure 7). PSTN $^{\text {Tac1 }}$ neurons uniquely projected to the PVT, PBN, NTS, and intermediate reticular nucleus (IRT), while PSTN ${ }^{\mathrm{CRH}}$ neurons uniquely projected to the lateral reticular nucleus (LRN). Both populations projected to the BNST, CeA, medial reticular nucleus (MRN), and tegmental reticular nucleus (TRN).

Because stimulation of PSTN ${ }^{\text {Tac1 }}$ neurons decreased feeding, we tested the effects of stimulating their projections to neuronal populations known to regulate feeding behavior. Optogenetic stimulation of projections from PSTN ${ }^{\text {Tac1 }}$ neurons to the BNST did not cause changes in food intake (Figures 8A-D). However, stimulation of projections from PSTN ${ }^{T a c 1}$ neurons to the CeA (Figures 8E-H), PVT (Figures 8I-L), PBN (Figures 8M-P), and NTS (Figures 8Q-T) caused decreases in food consumption during the stimulation period, demonstrating the sufficiency of activity in these projection targets for the cessation of food intake. 


\section{Discussion}

Taken together, our results show that the PSTN can be subdivided into two nearly distinct subpopulations (Figure 2). Both PSTN ${ }^{\mathrm{Tacl}}$ and PSTN ${ }^{\mathrm{CRH}}$ neurons increase activity in response to food exposure and administration of anorexigenic hormones (Figures 3 and 4). PSTN ${ }^{\mathrm{Tac} 1}$ neurons, but not PSTN ${ }^{\mathrm{CRH}}$ neurons, are necessary for the full anorexigenic effects of these hormones (Figure 5). Additionally, optogenetic or chemogenetic stimulation of PSTN ${ }^{\text {Tac1 }}$ neurons, but not PSTN ${ }^{\mathrm{CRH}}$ neurons, is sufficient to reduce food consumption (Figure 6). Consistently, these two populations have differential expression patterns, with PSTN ${ }^{\mathrm{Tac} 1}$ neurons uniquely projecting to downstream regions that suppress appetite including the PBN, NTS, and PVT (Figure 7). Stimulation of projections to these downstream regions is also sufficient to reduce food consumption (Figure 8). Taken together, these anatomical and functional results demonstrate that activity in PSTN ${ }^{\mathrm{Tacl}}$ neurons negatively regulates food consumption and identify multiple neural pathways through which they exert these appetite-suppressing effects.

\section{Effects of stimulation of PSTN neurons}

Stimulation of PSTN ${ }^{\text {Tacl }}$ neurons decreased food intake but did not eliminate consumption altogether. These effects are comparable to the reported effects of stimulating hypothalamic POMC neurons, which require several hours of stimulation for a statistically significant decrease in food intake to occur (Aponte et al., 2011), or the effects of stimulating PBN ${ }^{\text {CGRP }}$ neurons, which cause an immediate but not absolute reduction in feeding (Carter et al., 2013). Because PSTN $^{\mathrm{Tac1}}$ neurons are glutamatergic (Figures 2D and E), they likely decrease feeding by increasing activity in downstream anorexigenic neural populations. In addition to glutamatergic stimulation, tachykinin-1 is alternatively spliced into four neuropeptides including substance $\mathrm{P}$, 
which has been shown to suppress feeding when centrally administered in rodents and birds (Dib, 1999; Pauliukonis et al., 2020; Tachibana et al., 2010).

PSTN $^{\mathrm{CRH}}$ neurons exhibited an increase in neural activity following exposure to food stimuli, but stimulation or inhibition of these neurons caused no noticeable effects on food intake behavior. Therefore, these neurons may be involved in aspects of food intake not studied here, such as regulating nutrient intake or responding to food palatability. Interestingly, Zhu et al. found that deficiency of indispensable amino acids caused substantial upregulation of $\mathrm{Crh}$ transcript in PSTN ${ }^{\mathrm{CRH}}$ neurons (Zhu et al., 2012), suggesting that these neurons may mediate appetite for specific nutrients. Alternatively, these neurons may regulate conditioned associations between specific foods and aversive or appetitive cues.

Previous studies identified downstream projections from PSTN neurons to other anorexigenic neural populations (Barbier et al., 2020; Zhang and van den Pol, 2017), and our present results distinguish between projections from PSTN $^{\mathrm{Tac} 1}$ and PSTN ${ }^{\mathrm{CRH}}$ neurons. We initially identified the PSTN as a potential anorexigenic population by determining monosynaptic sources of input to PBN ${ }^{\text {CGRP }}$ neurons (Figure 1), subsequently determining that these projections originate from $\mathrm{PSTN}^{\mathrm{Tac} 1}$ neurons. Consistently, stimulation of these projections is sufficient to reduce food consumption (Figures 8M-P). Zhang et al. previously showed that stimulation of glutamatergic projections from the PSTN to the PVT was sufficient to reduce food consumption (Zhang and van den Pol, 2017), and our consistent results (Figures 8I-L) suggest that these projections originate from $\mathrm{PSTN}^{\mathrm{Tac} 1}$ neurons. We also determined that stimulation of projections from PSTN ${ }^{\text {Tac1 }}$ neurons to the CeA (Figures 8E-H) and NTS (Figures 8Q-T) reduce food consumption. Because we do not yet know whether individual PSTN ${ }^{\mathrm{Tac} 1}$ neurons project to one or more downstream regions, we cannot rule out the potential effects of antidromic 
activation of each projection target, although stimulation of projections to the BNST caused no observable effects.

\section{Role of PSTN neurons in mediating anorexigenic hormones}

In addition to food stimuli, we found that administration of the anorexigenic hormones amylin, CCK, and PYY caused activation of PSTN ${ }^{\mathrm{Tac} 1}$ and PSTN ${ }^{\mathrm{CRH}}$ neurons using Fos expression analysis (Figures 4A-E). Likewise, using fiber photometry, we observed a transient increase in activity in $\mathrm{PSTN}^{\mathrm{Tac} 1}$ neurons following administration of these hormones via an intraperitoneal catheter (Figures 4F-L). Interestingly, chemogenetic inhibition of $\operatorname{PSTN}^{\mathrm{Tac} 1}$ neurons attenuated but did not completely rescue the reduction in food intake caused by these hormones (Figures 5A-J), indicating that PSTN ${ }^{\text {Tac1 }}$ neuron activity is partially necessary for the appetite-suppressing effects of these hormones. These results are similar to the effects of inhibiting PBN ${ }^{\mathrm{CGRP}}$ neurons, which also attenuates but does not completely rescue decreases in food consumption following administration of anorexigenic compounds (Campos et al., 2016; Carter et al., 2013). It is interesting that chemogenetic inhibition of $\mathrm{PSTN}^{\mathrm{Tac} 1}$ neurons caused an increase in food intake behavior over a sustained period even though the recorded calcium dynamics in these neurons occurred over a relatively short period (Figures 4G-L). Perhaps the neuropeptides released by PSTN $^{\text {Tac1 }}$ neurons cause a sustained change in neural activity in downstream neural populations. Alternatively, perhaps $\operatorname{PSTN}^{\mathrm{Tac} 1}$ neurons exhibit a transient increase in activity but do not completely return to baseline levels over a sustained period in a way that is not detectable by fiber photometry. Indeed, comparisons of fiber photometry versus electrophysiology recordings of other neuronal populations, such as AgRP neurons (Chen et al., 2015; Mandelblat-Cerf et al., 
2015), demonstrate that increased activity of neurons may persist even when fiber photometry recordings return to baseline.

The necessity of the PSTN for the full suppression of appetite following administration of anorexigenic hormones is similar to the findings of Barbier et al., who report that PSTN neurons increase Fos expression in response to novel food exposure or to administration of the anorexigenic compound lipopolysaccharide (LPS) or the anticancer chemotherapy drug cisplatin (Barbier et al., 2020). Interestingly, chemogenetic inhibition of PSTN ${ }^{\text {Tacl }}$ neurons also attenuated but did not completely rescue the reduction in food intake caused by LPS. Inhibition also reduced the reduction of food consumption following exposure to a novel taste. Thus, the PSTN seems to be partially necessary for the suppression of food intake following a wide variety of stimuli including endogenous anorexigenic hormones, exogenous compounds that induce sickness, and behaviors such as taste neophobia. Interestingly, these stimuli all cause activation of neurons in regions downstream of the PSTN including the CeA and PBN. However, it is important to note that other studies show that PSTN neurons do not express Fos following administration of $\mathrm{LiCl}$, a compound that causes transient visceral malaise, nor sensory exposure to the bitter tastant quinine hydrochloride (Yasoshima et al., 2006). Future studies should examine a diverse range of stimuli that activate these neurons, as well as the necessity of each downstream projection in mediating their effects. 


\section{Methods}

\section{Animals}

All experiments were approved by the Institutional Animal Care and Use Committee at Williams College and were performed in accordance with the guidelines described in the U.S. National Institutes of Health Guide for the Care and Use of Laboratory Animals. We used Tac1 ${ }^{\text {Cre/+ }}$ mice (Harris et al., 2014) (Jackson Labs, \#021877), Crh ${ }^{C r e /+}$ mice (Taniguchi et al., 2011) (Jackson Labs, \#012704), and Calca ${ }^{\text {Cre/+ }}$ mice (Carter et al., 2013) (Jackson Labs, \#033168) bred on a C57B1/6 background. Each experimental group was composed of a randomized selection of mice with identical sex ratios and ages. To comply with NIH guidelines for using both sexes of animals (Clayton and Collins, 2014), we used an equal number of male and female animals across data sets. All mice were 7-9 weeks old at the time of surgery and no more than 16-20 weeks old at the cessation of experiments. During experimental procedures, mice were housed in

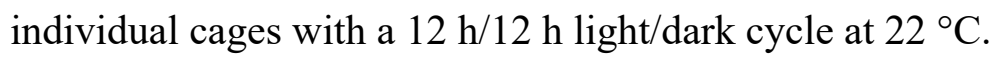

\section{Virus preparation}

Cre-inducible recombinant adeno-associated virus (AAV) vectors carrying mCherry (AAV9hSyn-DIO-mCherry, \#50459), GCaMP6s (AAV9-CAG-Flex-GCaMP6s, \#100842), hM3DqmCherry (AAV9-hSyn-DIO-hM3Dq-mCherry, \#44361), and hM4Di-mCherry (AAV9-hSynDIO-hM4Di-mCherry, \#44362) were obtained from Addgene. Cre-inducible AAV carrying ChR2-mCherry (AAV5-Ef1a-DIO-ChR2-mCherry, \#AV4379J) was obtained from the Vector Core at the University of North Carolina at Chapel Hill. Cre-inducible AAV carrying TVAmCherry (AAV5-EF1a-Flex-TVA-mCherry, \#GVVC-AAV-67) and rabies glycoprotein (RG, AAV8-CAG-Flex-RabiesG, \#GVVC-AAV-59) were obtained from the Neuroscience Gene 
Vector and Virus Core at Stanford University. Modified rabies virus carrying $\Delta$ G-eGFP (SAD$\Delta$ G-eGFP) was obtained from the Viral Vector Core of the Salk Institute. Viral aliquots were stored at $-80^{\circ} \mathrm{C}$ before stereotaxic injection.

\section{Stereotaxic surgery}

Mice were anaesthetized with 4\% isoflurane (Henry Schein Animal Health) and placed on a stereotaxic frame (David Kopf Instruments). Once on the frame and throughout the remainder of surgical procedures, mice received 1-2\% isoflurane trans-nasally. The skull was exposed and leveled in the horizontal plane. For viral targeting of transgenes to the PSTN, AAV was stereotaxically injected unilaterally or bilaterally, as described in the text, into the PSTN [anteroposterior (AP), $-2.4 \mathrm{~mm}$; mediolateral (ML), $\pm 1.1 \mathrm{~mm}$; dorsoventral (DV), $-5.25 \mathrm{~mm}$ ]. A total of $0.5 \mu \mathrm{l}$ of virus was injected at a rate of $0.1 \mu \mathrm{l} / \mathrm{min}$ and was allowed $8-10 \mathrm{~min}$ to diffuse before the injection needle was removed. For modified rabies retrograde labeling in the PBN, AAV Flex TVA-mCherry and AAV DIO RG vectors were unilaterally injected into the PBN (AP, $-4.9 \mathrm{~mm}$; ML, $1.4 \mathrm{~mm}$; DV, $3.8 \mathrm{~mm}$ ); two weeks later, SAD $\Delta \mathrm{G}$-eGFP was injected into the same location.

Following viral injection, mice used for fiber photometry experiments received unilateral surgical implantation of a mono fiber-optic cannula (Doric Lenses) above the PSTN (AP, -2.4 $\mathrm{mm}$; ML, $1.1 \mathrm{~mm}$; DV, $-4.85 \mathrm{~mm}$ ). Mice used for optogenetic experiments likewise received unilateral surgical implantation of a mono fiber-optic cannula above the PSTN (AP, $-2.4 \mathrm{~mm}$; ML, $1.1 \mathrm{~mm}$; DV, $-4.85 \mathrm{~mm}$ ), the NTS (AP, $-7.0 \mathrm{~mm}$; ML, $1.0 \mathrm{~mm}$; DV, $-3.8 \mathrm{~mm}$ ), the PBN (AP, $-5.2 \mathrm{~mm}$; ML, $1.6 \mathrm{~mm}$; DV, $-3.0 \mathrm{~mm}$ ), the PVT (AP, $-1.2 \mathrm{~mm}$; ML, $0.1 \mathrm{~mm}$; DV , -2.6 $\mathrm{mm}$ ), the CeA (AP, $-1.2 \mathrm{~mm}$; ML, $2.4 \mathrm{~mm}$; DV, $-4.0 \mathrm{~mm}$ ), or the BNST (AP, $0.1 \mathrm{~mm}$; ML, 0.8 
$\mathrm{mm} ; \mathrm{DV},-4.5 \mathrm{~mm})$. The cannulae were fixed onto the skull with C\&B Metabond (Parkell) and dental acrylic.

All mice were allowed at least 14 days to recover from surgery before the start of experimental procedures. Following behavioral experiments, brain sections containing the PSTN or PBN were examined for expression of virus and proper implantation of fiber-optic cannulae. Animals that did not show viral expression (mCherry or eGFP fluorescence) or proper cannulae placement were not included in subsequent data analysis. For anterograde and retrograde labelling experiments, animals were perfused at least two or three weeks after injection, respectively, to allow for maximal viral expression of fluorescent transgenes.

\section{Intraperitoneal catheter surgery}

Two weeks following stereotaxic injection of virus, mice used for fiber photometry experiments following hormone administration received surgical implantation of an intraperitoneal catheter. Catheters were made from hollow polyurethane tubing $(0.015$ x 1.043 inches; Instech, BTPE-20) and sterilized $24 \mathrm{~h}$ prior to surgery. Mice were anesthetized with $2.5 \%$ isoflurane (Henry Schein Animal Health) through a nose cone. Hair was removed from the animal's ventral abdomen and dorsal back near the scapulae using Nair Hair Remover. A small incision was made between the scapulae and the skin was bluntly dissected from the subcutaneous tissue toward the left flank. A transverse abdominal skin incision was made and the skin was bluntly dissected from the subcutaneous tissue toward the flank to complete a subcutaneous tunnel between the two skin incisions. A sterilized catheter attached to a button (Instech, \#1-VABM1B/25) was pulled through the tunnel using a hemostat. A small incision was made into the abdominal cavity and the tip of the catheter was placed into the incision site and sutured into place. The button was 
inserted into the incision made between the scapulae under the skin and was secured with sutures. The absence of leakage was confirmed by injecting $0.9 \%$ saline into the catheter and looking for liquid on the outside of the mouse. The abdominal muscle was sutured and the skin incision was closed in two layers. A magnetic cap (Instech, \#1-VABM1C) was placed onto the button to prevent foreign materials from entering the intraperitoneal cavity through the button. Animals were allowed another 14 days to recover from surgery before the start of infusion experiments.

\section{Food intake measurements}

For feeding assays, mice were individually housed in specialized food/liquid intake measurement cages attached to water bottles mounted on scales (DietMax, Omnitech Electronics). Mice were provided a liquid diet of Vanilla Ensure (Abbott Laboratories) diluted in a 1:1 ratio with water for a total caloric density of $450 \mathrm{kcal} / \mathrm{L}$. Bottles containing liquid diet were washed and disinfected daily and fully replenished at the beginning of the light cycle. Mice were also provided ad libitum access to HydroGel $\left(\mathrm{ClearH}_{2} \mathrm{O}\right.$, \#70-01-5022) to ensure constant hydration. Individual feeding bouts were recorded using scale measurements (Fusion Software, Omnitech Electronics). A meal was defined as any linear decrease greater than $0.02 \mathrm{~g}$ of liquid Ensure. The minimum time between two meals was $15 \mathrm{~s}$ (otherwise, only one meal was recorded). Mice were allowed to habituate for a minimum of $72 \mathrm{~h}$ prior to the beginning of experiments, and all food intake measurements were obtained during the middle $4 \mathrm{~h}$ of the inactive cycle. 


\section{Pharmacology}

All compounds were prepared in $0.9 \%$ sterile saline (VWR, \#100216) and stored at $-20{ }^{\circ} \mathrm{C}$ before use. Compounds consisted of $0.9 \%$ sterile saline, amylin (10 $\mu \mathrm{g} / \mathrm{kg}$; Bachem, \#H-9475),

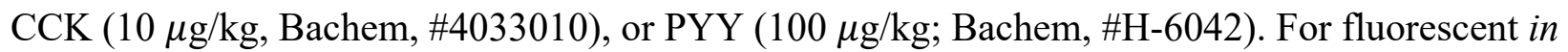
situ hybridization experiments, these compounds were injected intraperitoneally using a 25G syringe 45 min before anesthesia and perfusion. For chemogenetic experiments, mice received intraperitoneal injection of clozapine-N-oxide (CNO; $0.3 \mathrm{mg} / \mathrm{kg}$, Sigma-Aldrich, \#C0832) 10 min prior to injection of an anorexigenic compound followed immediately by food intake recordings. Each mouse was used for a total of 5 experimental sessions, and the mean food intake value for each mouse was included for data analysis across all mice within an experimental group.

\section{Fiber photometry}

All fiber photometry experiments were performed in clear circular chambers with fresh bedding for each trial. Mice implanted with mono fiber-optic cannulae were attached to optical patchcords (400 $\mu \mathrm{m}$ core, $0.48 \mathrm{NA}, 1 \mathrm{~m}$ long; Doric Lenses) via zirconia connectors (Doric Lenses, Sleeve_ZR_2.5_BK) at least 30 min before each trial to allow for habituation.

During each trial, a GCaMP excitation wavelength of $465 \mathrm{~nm}$ blue light modulated at 566 $\mathrm{Hz}$ was delivered through the patchcord. A control wavelength of $405 \mathrm{~nm}$ violet light modulated at $211 \mathrm{~Hz}$ was also delivered to detect calcium-independent GCaMP fluorescence or photobleaching. Delivered frequencies were offset to mitigate contamination or interference from electrical noise in the testing room. Excitation and control lights were generated from light emitting diodes (LEDs; Tucker-Davis Technologies, CLED_465 and CLED_405) and processed 
through a real-time amplifier (Tucker-Davis Technologies, RZ5P). Fluorescence signals were detected by a visible femtowatt photoreceiver (Tucker-Davis Technologies, Model 2151) with gain set to DC low. The light was then converted to electrical signals and demodulated by a realtime processor (Tucker-Davis Technologies, RZ5P). Data were recorded using Synapse software (Tucker-Davis Technologies).

To test the effects of food exposure on PSTN activity, mice were either fasted for $18 \mathrm{~h}$ or fed ad libitum. After acclimating to the cage, a 30-min baseline recording was produced to ensure a stable signal and decrease photobleaching. At time $\mathrm{t}=0$, mice were provided with either standard laboratory chow pellets, a small scoop of peanut butter, or a novel object (a mini screwdriver or roll of tape). To eliminate any effects of novelty, mice were provided peanut butter in their home cages for at least 2 days prior to testing. In some experiments, mice were water-deprived for $16 \mathrm{~h}$ and at time $\mathrm{t}=0$ a water port was placed in the cage.

To test the effects of peripheral hormone administration, mice were food deprived for 18 $\mathrm{h}$ prior to acclimating in the testing chamber. Amylin, CCK, PYY, or 0.9\% saline was delivered by intraperitoneal catheter at a total volume of $300 \mu$ l. Following hormone injection, the photometry recording continued for $20 \mathrm{~min}$.

Data were analyzed using custom MATLAB (MathWorks) scripts. The 465 and 405 signals were independently downsampled to $1 \mathrm{~Hz}$ and normalized to baseline signals to determine $\Delta \mathrm{F} / \mathrm{F}$, in which $\Delta \mathrm{F} / \mathrm{F}=\left(\mathrm{F}-\mathrm{F}_{\text {baseline }}\right) / \mathrm{F}_{\text {baseline, }}$ and $\mathrm{F}_{\text {baseline }}$ is the median of $30 \mathrm{~s}$ baseline recording prior to time zero. No isosbestic normalization was introduced. To eliminate movement and bleaching artifacts, recordings with more than $20 \%$ change in the $405 \mathrm{~nm}$ signal were excluded from analyses. Plots representing mean \pm standard error $\Delta \mathrm{F} / \mathrm{F}$ signals for each experiment and heatmaps representing $\Delta \mathrm{F} / \mathrm{F}$ for each trial were generated in MATLAB. 
Quantitative data analysis was performed in MATLAB and Prism 8.0 (GraphPad). The maximum $\Delta \mathrm{F} / \mathrm{F}$ was defined as the maximum $\Delta \mathrm{F} / \mathrm{F}$ signal intensity from $\mathrm{t}=0$ to $\mathrm{t}=75 \mathrm{~s}$. The area under the curve was defined as the integral of $\Delta \mathrm{F} / \mathrm{F}$ from $\mathrm{t}=0$ until the time when $\Delta \mathrm{F} / \mathrm{F}$ returned to 0 .

\section{Optogenetic photostimulation}

Mice were attached to fiber optic cables (200 $\mu \mathrm{m}$ core, 0.2 NA, $2 \mathrm{~m}$ long; Doric Lenses), coated with opaque heat-shrink tubing, via zirconia connectors (Doric Lenses, Sleeve_ZR_2.5) and allowed to acclimate for at least $3 \mathrm{~d}$ prior to experimental sessions. Cables were attached to a 473 nm blue-light laser (LaserGlow) driven by a Master-8 Pulse Stimulator (A.M.P.I.). Light was delivered in $10 \mathrm{~ms}$ pulses at $20 \mathrm{~Hz}$ for $1 \mathrm{~s}$ every $4 \mathrm{~s}$ over a 1 -h period. During the testing period, food intake was measured for $1 \mathrm{~h}$ before photostimulation, $1 \mathrm{~h}$ during photostimulation, and $1 \mathrm{~h}$ after photostimulation. Each mouse was used for a total of 5 experimental sessions, and the mean food intake value for each mouse was included for data analysis across all mice within an experimental group.

\section{Perfusions and sectioning}

Mice were anesthetized with intraperitoneal injection of 2, 2, 2-Tribromoethanol (SigmaAldrich, \#48402) dissolved in Tert-amyl alcohol and sterile $0.9 \%$ saline. Mice were then transcardially perfused with cold $0.01 \mathrm{M}$ phosphate buffered saline (PBS), pH 7.4, followed by 4\% paraformaldehyde in PBS. The brains were extracted, allowed to postfix overnight in 4\% paraformaldehyde at $4{ }^{\circ} \mathrm{C}$, and cryoprotected in $30 \%$ sucrose dissolved in PBS for an additional $24 \mathrm{~h}$ at $4{ }^{\circ} \mathrm{C}$. Each brain was sectioned coronally at $30 \mu \mathrm{m}$ on a microtome (Leica Microsystems) 
and collected in cold PBS. For projection tracing and fluorescent in situ hybridization experiments, the left side of each brain was marked with a pinhole to ensure images were taken from the same hemisphere in each brain. Brain sections were mounted onto SuperFrost Plus glass slides (VWR, \#48311-703) and either immediately used for in situ hybridization experiments, immunohistochemistry experiments, or coverslipped with DAPI Fluoromount-G (Southern Biotech, \#0100-20) and stored in the dark at $4{ }^{\circ} \mathrm{C}$.

\section{Fluorescent in situ hybridization}

Fluorescent in situ hybridization reactions were performed using an RNAscope Multiplex Fluorescent Reagent Kit v2 (ACDBio, \#323100) according to the manufacturer's instructions. Brain sections mounted onto SuperFrost Plus glass slides (VWR, \#48311-703) were labelled using a combination of target probes for Crh (Probe-Mm-Crh, \#316091), Tac1 (Probe-Mm-Tac1, \#410351; or Probe-Mm-Tac1-C3, \#410351-C3), Slc17a6 (Probe-Mm-Slc17a6-C2, \#319171-C2), Calb1 (Probe-Mm-Calb1-C2, \#428431-C2), Calb2 (Probe-Mm-Calb2-C2, \#313641), Pvalb (Probe-Mm-Pvalb-C2, \#4219310-C2), and Fos (Probe-Mm-Fos-C2; \#316921-C2). The Fos probe was diluted 1:10 to reduce background hybridization. Fluorophore reagent packs (Akoya Biosciences) including Opal 520 (FP1487001KT), Opal 570 (FP1488001KT), and Opal 690 (FP1497001KT) were diluted to a final concentration of 1:1500 with TSA buffer. Following staining procedures, slides were coverslipped with Fluoromount-G (Southern Biotech, \#0100-01) and stored in the dark at $4{ }^{\circ} \mathrm{C}$ until microscopy and imaging. 


\section{Immunohistochemistry}

Brain sections were washed three times in PBS with $0.2 \%$ Triton X-100 (PBST) for 10 min at room temperature. Sections were then incubated in a blocking solution composed of PBST with 3\% normal donkey serum (Jackson ImmunoResearch, \#017-000-121) for $15 \mathrm{~min}$ at room temperature. For primary antibody exposure, sections were incubated in rabbit anti-Fos (1:1000; Cell Signaling Technology, \#2250) in blocking solution overnight at $4{ }^{\circ} \mathrm{C}$. After three 5 min washes in blocking solution, sections were incubated in AlexaFluor 488 donkey anti-rabbit (1:250; Jackson ImmunoResearch, \#711-545-152) in block solution for $1 \mathrm{~h}$ at room temperature. Finally, sections were washed three times in PBS. Following staining procedures, slides were coverslipped with DAPI Fluoromount-G (Southern Biotech, \#0100-20) and stored in the dark at $4{ }^{\circ} \mathrm{C}$ until microscopy and imaging.

\section{Microscopy}

For confirmation of virally targeted reporter expression, analysis of anterograde/retrograde projections, and examination of immunofluorescent labelling, slides were examined using an Eclipse 80i epifluorescent microscope (Nikon) and images were captured using a Retiga 2000R digital camera (QImaging). For FISH experiments, slides were examined using a Leica DMi8 confocal microscope. Single channel and overlay pictures were acquired for each section. The resulting images were minimally processed using Photoshop (Adobe) to enhance the brightness and contrast for optimal representation of the data. All digital images were processed in the same way between experimental conditions to avoid artificial manipulation between different datasets.

Analysis of anterograde or retrograde expression was performed on coronal brain sections spanning the entire rostrocaudal distance of the brain. Fluorescently labelled brain 
regions were identified using the Allen Mouse Brain Atlas (Dong, 2008) and the Paxinos and Franklin Mouse Brain Atlas (Franklin and Paxinos, 2013).

Quantification of colocalization of fluorescently-labelled markers in the PSTN was performed on adjacent sections from $\sim-2.3$ to $-2.84 \mathrm{~mm}$ from bregma (18 sections per mouse). The Photoshop count tool was used to accurately identify and tally individual neurons. Cell counts were corrected for potential double counting using Abercrombie's formula (Guillery, 2002).

\section{Experimental design and statistical analysis}

We used a between-subjects experimental design for all experiments. To determine an effective sample size for statistical comparisons, we used an online power and sample size calculator (https://clincalc.com/stats/samplesize.aspx). Assuming a significance level of 0.05, this calculator shows that with at least four mice per group, we had an $80 \%$ confidence level of achieving statistical significance between means of 1.1-fold. We excluded an animal from data analysis if flagged by an animal care technician for health reasons during the experimental period or if post hoc histological analysis showed no viral transduction as indicated by an absence of mCherry or GCaMP fluorescence.

Data were analyzed using Prism 8.0 (GraphPad Software). Statistical tests included oneway ANOVA (Figure 4E), one-way ANOVA with repeated measures (Figures 3K and L; 4K-L), two-way ANOVA (Figure 3C), two-way ANOVA with repeated measures (Figures 5D-N; 6D and $\mathrm{E} ; 6 \mathrm{~K}$ and $\mathrm{L} ; 8 \mathrm{C}$ and $\mathrm{D} ; 8 \mathrm{G}$ and $\mathrm{H} ; 8 \mathrm{~K}$ and $\mathrm{L} ; 8 \mathrm{O}$ and $\mathrm{P} ; 8 \mathrm{~S}$ and $\mathrm{T}$ ), and unpaired two-tailed $t$-test (Figure 3D), as described in the text and Supplementary Table 1. Graphs were exported from Prism 8.0 to Illustrator (Adobe) for preparation of figures. 


\section{Figure Legends:}

Figure 1. PSTN neurons project to anorexigenic PBNCGRP neurons and are activated by refeeding.

(A) Modified rabies viral strategy used to identify afferent input to PBN ${ }^{C G R P}$ neurons.

(B) Confirmation of modified rabies virus reporter transgenes in PBN ${ }^{\text {CGRP }}$ neurons. Scale bar, $250 \mu \mathrm{m}$.

(C) Sagittal mouse brain diagram showing locations of retrograde eGFP expression in (D-H).

(D-H) eGFP-expressing neurons in (D) the bed nucleus of the stria terminalis (BNST); (E) the central nucleus of the amygdala (CeA); (F) the hypothalamic arcuate nucleus (Arc); (G) the parasubthalamic nucleus (PSTN); and (H) the nucleus of the solitary tract (NTS). Scale bar, 250 $\mu \mathrm{m}$.

(I) Coronal mouse brain diagram depicting the bilateral location of the PSTN medial to the cerebral peduncle (cp). Blue arrows point to the location of the PSTN.

(J) Immunolabelling of Fos expression following 18-h food deprivation. Scale bar, $500 \mu \mathrm{m}$. (K) Immunolabelling of Fos expression following 18-h food deprivation followed by 30 min refeeding. Right, higher magnification image.

\section{Figure 2. Characterization of PSTN cell types.}

(A) In situ hybridization images of selected mRNA transcripts from the Allen Mouse Brain Expression Atlas. cp, cerebral peduncle. Scale bar, $500 \mu \mathrm{m}$.

(B) Two-color fluorescent in situ hybridization shows near distinct expression of Tacl and Crh in PSTN neurons. Right, higher magnification image. Scale bar, $200 \mu \mathrm{m}$.

(C) Quantification of overlap between $\operatorname{PSTN}^{\mathrm{Tac} 1}$ and PSTN ${ }^{\mathrm{CRH}}$ neurons. 
(D-K) Three-color fluorescent in situ hybridization comparing spatial overlap between neurons expressing Tac1 and Crh with (D,E) Slc17a6; (F,G) Calb1; (H,I) Calb2; and (J,K) Pvalb. Insets show higher magnification images from middle column. Data at right represent mean \pm standard deviation. Dots represent individual experimental animals. Scale bar, $100 \mu \mathrm{m}$.

\section{Figure 3. Refeeding and food consumption activate PSTN neurons.}

(A-B) Three-color fluorescent in situ hybridization comparing co-expression of Fos in neurons expressing Tacl or Crh following (A) 18-h food deprivation or (B) 18-h food deprivation followed by 30 min refeeding. Insets show higher magnification images from middle column. Scale bar, $200 \mu \mathrm{m}$.

(C) Quantification of the percentage of Tacl- or Crh-expressing neurons co-expressing Fos in 18-h food deprivation versus refeeding conditions.

(D) Quantification of the number of Tac1- or Crh-expressing neurons co-expressing Fos following refeeding.

(E) Diagrams showing (left) viral injection strategy to unilaterally target PSTN $^{\text {Tac1 }}$ neurons with GCaMP6s and (right) subsequent fiber photometry recording in freely moving mice.

(F) Representative photomicrograph showing PSTN ${ }^{\text {Tac1 }}$ neurons expressing GCaMP6s. Scale bar, $100 \mu \mathrm{m}$.

(G-J) Top, fiber photometry traces in $\operatorname{PSTN}^{\text {Tac1 }}$ neurons following exposure to (G) standard mouse chow, $(\mathrm{H})$ peanut butter, or $(\mathrm{I})$ a novel object from animals food deprived for $18 \mathrm{~h}$ (magenta) or fed ad libitum (grey). (J) Fiber photometry trace in PSTN ${ }^{\mathrm{Tac} 1}$ neurons following exposure to a water port in water-deprived mice. Data represent the mean \pm standard error. 
Vertical dashed lines depict time of exposure. Bottom, heat maps depicting changes in fluorescence intensity in individual animals.

(K) Quantification of maximum values of fluorescence intensity in conditions (G-J).

(L) Quantification of area under the curve of fluorescence intensity among conditions in (G-J).

Data represent mean \pm standard error. Dots represent individual experimental animals. Post hoc comparisons: ${ }^{* *} \mathrm{p}<0.01,{ }^{* * *} \mathrm{p}<0.001, * * * * \mathrm{p}<0.0001$. See Supplementary Table 1 for additional statistical information.

\section{Figure 4. Anorexigenic hormones cause transient activation of PSTN neurons.}

(A-D) Three-color fluorescent in situ hybridization comparing co-expression of Fos in neurons expressing Tacl or Crh following administration of (A) saline, (B) amylin, (C) CCK, or (D) PYY. Insets show higher magnification images from middle column. Scale bar, $200 \mu \mathrm{m}$.

(E) Quantification of the percentage of Tacl- or Crh-expressing neurons co-expressing Fos across conditions in (A-D).

(F) Diagram showing (left) viral injection strategy to unilaterally target $\operatorname{PSTN}^{\mathrm{Tac}}$ neurons with GCaMP6s and (right) subsequent fiber photometry recording in freely moving mice coupled with intraperitoneal infusion of anorexigenic hormones.

(G-J) Top, fiber photometry traces in $\mathrm{PSTN}^{\mathrm{Tac} 1}$ neurons following intraperitoneal infusion of (G) saline, (H) amylin, (I) CCK, or (J) PYY. Data represent the mean \pm standard error. Vertical dashed lines depict time of injection. Bottom, heat maps depicting changes in fluorescence intensity in individual animals.

(K) Quantification of maximum values of fluorescence intensity in conditions (G-J).

(L) Quantification of area under the curve of fluorescence intensity among conditions in (G-J). 
Data represent mean \pm standard error. Dots represent individual experimental animals. Post hoc comparisons: ${ }^{*} \mathrm{p}<0.05, * * \mathrm{p}<0.01, * * * \mathrm{p}<0.001, * * * * \mathrm{p}<0.0001$. See Supplementary Table 1 for additional statistical information.

\section{Figure 5. Inhibition of PSTN ${ }^{\text {Tac1 }}$ neurons attenuates the anorexigenic effects of appetite-}

\section{suppressing hormones.}

(A) Diagram showing (left) viral injection strategy to unilaterally target PSTN ${ }^{\mathrm{Tac} 1}$ or PSTN ${ }^{\mathrm{CRH}}$ neurons with hM4Di-mCherry or mCherry transgenes and (right) subsequent food intake measurements following administration of $\mathrm{CNO}$ and anorexigenic hormones.

(B) Representative photomicrograph showing PSTN ${ }^{\text {Tac1 }}$ neurons expressing hM4Di-mCherry. Scale bar, $100 \mu \mathrm{m}$.

(C) Representative photomicrograph showing PSTN ${ }^{\mathrm{CRH}}$ neurons expressing hM4Di-mCherry.

(D-G) Cumulative food intake in $\mathrm{Tacl}^{\mathrm{Cre} /+}$ animals administered CNO followed with (D) saline, (E) amylin, (F) CCK, or (G) PYY.

(H-J) Quantification of (H) meal size, (I) meal duration, (J) and meal frequency in conditions (D-G).

(K-N) Cumulative food intake in $\mathrm{Crh}^{\mathrm{Cr} /+}$ animals administered CNO followed with (K) saline, (L) amylin, (M) CCK, or (N) PYY.

Data represent mean \pm standard error. Dots represent individual experimental animals. Post hoc comparisons: ${ }^{*} \mathrm{p}<0.05, * * \mathrm{p}<0.01, * * * \mathrm{p}<0.001, * * * * \mathrm{p}<0.0001$. See Supplementary Table 1 for additional statistical information. 


\section{Figure 6. Stimulation of PSTN ${ }^{\text {Tac1 }}$ neurons is sufficient to suppress feeding.}

(A) Diagram showing (top) viral injection strategy to unilaterally target PSTN ${ }^{\mathrm{Tac} 1}$ or PSTN ${ }^{\mathrm{CRH}}$ neurons with ChR2-mCherry or mCherry transgenes and (bottom) subsequent optogenetic manipulation during food intake recordings.

(B) Representative photomicrograph showing PSTN ${ }^{\mathrm{Tac} 1}$ neurons expressing ChR2-mCherry. Scale bar, $100 \mu \mathrm{m}$.

(C) Representative photomicrograph showing PSTN ${ }^{\mathrm{CRH}}$ neurons expressing ChR2-mCherry. (D-E) Quantification of (D) cumulative food intake and (E) total food consumed during prestimulation, stimulation, and post-stimulation of $\operatorname{PSTN}^{\mathrm{Tac} 1}$ neurons. Blue background represents the 60-min photostimulation period.

(F-G) Quantification of (F) cumulative food intake and (G) total food consumed during prestimulation, stimulation, and post-stimulation of PSTN ${ }^{\mathrm{CRH}}$ neurons. Blue background represents the 60-min photostimulation period.

(H) Diagram showing (top) viral injection strategy to unilaterally target PSTN $^{\text {Tac1 }}$ or PSTN ${ }^{C R H}$ neurons with hM3Dq-mCherry or mCherry transgenes and (bottom) subsequent chemogenetic manipulation during food intake recordings.

(I) Representative photomicrograph showing PSTN $^{\mathrm{Tac} 1}$ neurons expressing hM3Dq-mCherry. (J) Representative photomicrograph showing PSTN ${ }^{\mathrm{CRH}}$ neurons expressing hM3Dq-mCherry. (K-L) Quantification of cumulative food intake in (K) $\mathrm{Tacl}^{\mathrm{Cre} /+}$ or (L) $\mathrm{Crh}^{\mathrm{Cre} /+}$ mice following administration of $\mathrm{CNO}$. 
Data represent mean \pm standard error. Dots represent individual experimental animals. Post hoc comparisons: ${ }^{*} \mathrm{p}<0.05,{ }^{*} \mathrm{p}<0.01,{ }^{* * *} \mathrm{p}<0.001, * * * * \mathrm{p}<0.0001$. See Supplementary Table 1 for additional statistical information.

Fig. 7. Nonidentical efferent projections from PSTN ${ }^{\text {Tac1 }}$ and PSTN ${ }^{\text {CRH }}$ neurons throughout the brain.

(A-B) Left, diagram showing viral injection strategy to unilaterally target (A) $\operatorname{PSTN}^{\text {Tac1 }}$ or (B) PSTN $^{\mathrm{CRH}}$ neurons with the mCherry transgene. Right, representative images of mCherry expression. Scale bar, $100 \mu \mathrm{m}$.

(C-J) Representative images at (left) lower and (right) higher magnification of mCherry expression in PSTN ${ }^{\mathrm{Tac} 1}$ projections throughout the brain. Scale bars, $500 \mu \mathrm{m}$.

(K-O) Representative images at lower (left) and higher (right) magnification of mCherry expression in PSTN ${ }^{\mathrm{CRH}}$ projections. Scale bars, $500 \mu \mathrm{m}$.

(P) Sagittal mouse brain diagram summarizing locations of PSTN $^{\mathrm{Tac} 1}$ and $\mathrm{PSTN}^{\mathrm{CRH}}$ projections throughout the brain.

BNST, bed nucleus of the stria terminalis; CeA, central nucleus of the amygdala; IRN, intermediate reticular nucleus; LRN, lateral reticular nucleus; MRN, midbrain reticular nucleus; NTS, nucleus of the solitary tract; PBN, parabrachial nucleus; PVT, paraventricular thalamic nucleus; TRN, tegmental reticular nucleus.

\section{Figure 8. Stimulation of PSTN ${ }^{\text {Tac1 }}$ neuron projections is sufficient to suppress feeding.}

(A) Diagram showing optogenetic strategy to unilaterally target PSTN ${ }^{\mathrm{Tac} 1}$ neurons with ChR2mCherry or mCherry transgenes with ipsilateral optic fiber implantation above the BNST. 
(B) Representative photomicrograph showing PSTN $^{\mathrm{Tac} 1}$ projections expressing ChR2-mCherry in the BNST.

(C-D) Quantification of (C) cumulative food intake and (D) total food consumed during prestimulation, stimulation, and post-stimulation of $\operatorname{PSTN}^{\mathrm{Tac} 1}$ projections to the BNST.

(E) Diagram showing optogenetic strategy to unilaterally target PSTN ${ }^{\mathrm{Tac} 1}$ neurons with ChR2mCherry or mCherry transgenes with ipsilateral optic fiber implantation above the CeA.

(F) Representative photomicrograph showing PSTN ${ }^{\text {Tac1 }}$ projections expressing ChR2-mCherry in the CeA.

(G-H) Quantification of $(\mathrm{G})$ cumulative food intake and $(\mathrm{H})$ total food consumed during prestimulation, stimulation, and post-stimulation of $\operatorname{PSTN}^{\mathrm{Tac} 1}$ projections to the CeA.

(I) Diagram showing optogenetic strategy to unilaterally target $\mathrm{PSTN}^{\mathrm{Tac} 1}$ neurons with ChR2mCherry or mCherry transgenes with ipsilateral optic fiber implantation above the PVT.

(J) Representative photomicrograph showing $\operatorname{PSTN}^{\mathrm{Tac} 1}$ projections expressing ChR2-mCherry in the PVT.

(K-L) Quantification of (K) cumulative food intake and (L) total food consumed during prestimulation, stimulation, and post-stimulation of PSTN $^{\text {Tac1 }}$ projections to the PVT.

(M) Diagram showing optogenetic strategy to unilaterally target PSTN ${ }^{\mathrm{Tac} 1}$ neurons with ChR2mCherry or mCherry transgenes with ipsilateral optic fiber implantation above the PBN.

(N) Representative photomicrograph showing PSTN $^{\mathrm{Tac} 1}$ projections expressing ChR2-mCherry in the PBN.

(O-P) Quantification of $(\mathrm{O})$ cumulative food intake and $(\mathrm{P})$ total food consumed during prestimulation, stimulation, and post-stimulation of $\operatorname{PSTN}^{\mathrm{Tac} 1}$ projections to the PBN. 
(Q) Diagram showing optogenetic strategy to unilaterally target $\mathrm{PSTN}^{\mathrm{Tac} 1}$ neurons with ChR2mCherry or mCherry transgenes with ipsilateral optic fiber implantation above the NTS.

(R) Representative photomicrograph showing PSTN $^{\mathrm{Tac} 1}$ projections expressing ChR2-mCherry in the NTS.

(S-T) Quantification of (S) cumulative food intake and (T) total food consumed during prestimulation, stimulation, and post-stimulation of $\operatorname{PSTN}^{\mathrm{Tac} 1}$ projections to the NTS.

Data represent mean \pm standard error. Dots represent individual experimental animals. Blue background represents the 60 -min photostimulation period. Post hoc comparisons: ${ }^{*} \mathrm{p}<0.05$, $* * \mathrm{p}<0.01, * * * \mathrm{p}<0.001, * * * * \mathrm{p}<0.0001$. See Supplementary Table 1 for additional statistical information. Scale bar, $250 \mu \mathrm{m}$. 


\section{Acknowledgements}

This research is supported by NIH grant DK105510 from the National Institute of Digestive and

Diabetes and Kidney Diseases (NIDDK) and by National Science Foundation grant 1652060 to

M.E.C. We thank R. O'Sullivan for performing initial pilot experiments, S. Kanoski, E. Noble,

A. Cortella, and S.-Y. Kim for advice on RNAscope procedures, and J. Cone, S.C. Doret, N.

Goldstein, A. Alhadeff, and N. Betley for advice on fiber photometry.

\section{Author contributions}

M.E.C. conceived of and supervised the study. J.H.K., G.H.K., J.S., K.H., L.B.H., M.C.N.,

O.K.B., S.L., T.B.L., F.F.G., S.C.F., and M.E.C. performed experiments, analyzed data, and

prepared figures for publication. S.C.F. and M.J.K. performed modified rabies virus experiments.

K.E.J. and F.F.G. set up fiber photometry equipment, wrote original Matlab code, and performed

data analysis. M.E.C., J.H.K., and G.H.K. wrote the manuscript with feedback and revisions

from all coauthors. 


\section{References}

Andermann, M.L., and Lowell, B.B. (2017). Toward a Wiring Diagram Understanding of Appetite Control. Neuron 95, 757-778.

Aponte, Y., Atasoy, D., and Sternson, S.M. (2011). AGRP neurons are sufficient to orchestrate feeding behavior rapidly and without training. Nature neuroscience 14, 351-355.

Barbier, M., Chometton, S., Pautrat, A., Miguet-Alfonsi, C., Datiche, F., Gascuel, J., Fellmann, D., Peterschmitt, Y., Coizet, V., and Risold, P.Y. (2020). A basal ganglia-like corticalamygdalar-hypothalamic network mediates feeding behavior. Proc Natl Acad Sci U S A 117, 15967-15976.

Barbier, M., Chometton, S., Peterschmitt, Y., Fellmann, D., and Risold, P.Y. (2017).

Parasubthalamic and calbindin nuclei in the posterior lateral hypothalamus are the major hypothalamic targets for projections from the central and anterior basomedial nuclei of the amygdala. Brain Struct Funct.

Cai, H., Haubensak, W., Anthony, T.E., and Anderson, D.J. (2014). Central amygdala PKCdelta $(+)$ neurons mediate the influence of multiple anorexigenic signals. Nat Neurosci 17 , 1240-1248.

Campos, C.A., Bowen, A.J., Han, S., Wisse, B.E., Palmiter, R.D., and Schwartz, M.W. (2017). Cancer-induced anorexia and malaise are mediated by CGRP neurons in the parabrachial nucleus. Nat Neurosci 20, 934-942.

Campos, C.A., Bowen, A.J., Schwartz, M.W., and Palmiter, R.D. (2016). Parabrachial CGRP Neurons Control Meal Termination. Cell metabolism 23, 811-820.

Carter, M.E., Soden, M.E., Zweifel, L.S., and Palmiter, R.D. (2013). Genetic identification of a neural circuit that suppresses appetite. Nature 503, 111-114.

Chen, Y., Lin, Y.C., Kuo, T.W., and Knight, Z.A. (2015). Sensory detection of food rapidly modulates arcuate feeding circuits. Cell 160, 829-841.

Chometton, S., Pedron, S., Peterschmitt, Y., Van Waes, V., Fellmann, D., and Risold, P.Y. (2016). A premammillary lateral hypothalamic nuclear complex responds to hedonic but not aversive tastes in the male rat. Brain Struct Funct 221, 2183-2208.

Ciriello, J., Solano-Flores, L.P., Rosas-Arellano, M.P., Kirouac, G.J., and Babic, T. (2008). Medullary pathways mediating the parasubthalamic nucleus depressor response. Am J Physiol Regul Integr Comp Physiol 294, R1276-1284.

Clayton, J.A., and Collins, F.S. (2014). Policy: NIH to balance sex in cell and animal studies. Nature 509, 282-283.

Dib, B. (1999). Food and water intake suppression by intracerebroventricular administration of substance $\mathrm{P}$ in food- and water-deprived rats. Brain Res 830, 38-42.

Dong, H.W. (2008). Allen reference atlas : a digital color brain atlas of the C57Black/6J male mouse (Hoboken, N.J.: Wiley).

Essner, R.A., Smith, A.G., Jamnik, A.A., Ryba, A.R., Trutner, Z.D., and Carter, M.E. (2017). AgRP Neurons Can Increase Food Intake during Conditions of Appetite Suppression and Inhibit Anorexigenic Parabrachial Neurons. J Neurosci 37, 8678-8687.

Franklin, K.B.J., and Paxinos, G. (2013). Paxinos and Franklin's The mouse brain in stereotaxic coordinates, Fourth edition. edn (Amsterdam: Academic Press, an imprint of Elsevier).

Goto, M., and Swanson, L.W. (2004). Axonal projections from the parasubthalamic nucleus. J Comp Neurol 469, 581-607.

Guillery, R.W. (2002). On counting and counting errors. J Comp Neurol 447, 1-7. 
Harris, J.A., Hirokawa, K.E., Sorensen, S.A., Gu, H., Mills, M., Ng, L.L., Bohn, P., Mortrud, M., Ouellette, B., Kidney, J., et al. (2014). Anatomical characterization of Cre driver mice for neural circuit mapping and manipulation. Front Neural Circuits 8, 76.

Holt, M.K., Pomeranz, L.E., Beier, K.T., Reimann, F., Gribble, F.M., and Rinaman, L. (2019). Synaptic Inputs to the Mouse Dorsal Vagal Complex and Its Resident Preproglucagon Neurons. J Neurosci 39, 9767-9781.

Huang, D., Grady, F.S., Peltekian, L., Laing, J.J., and Geerling, J.C. (2021). Efferent projections of CGRP/Calca-expressing parabrachial neurons in mice. J Comp Neurol 529, 2911-2957.

Krashes, M.J., Koda, S., Ye, C., Rogan, S.C., Adams, A.C., Cusher, D.S., Maratos-Flier, E., Roth, B.L., and Lowell, B.B. (2011). Rapid, reversible activation of AgRP neurons drives feeding behavior in mice. J Clin Invest 121, 1424-1428.

Lein, E.S., Hawrylycz, M.J., Ao, N., Ayres, M., Bensinger, A., Bernard, A., Boe, A.F., Boguski, M.S., Brockway, K.S., Byrnes, E.J., et al. (2007). Genome-wide atlas of gene expression in the adult mouse brain. Nature 445, 168-176.

Livneh, Y., Ramesh, R.N., Burgess, C.R., Levandowski, K.M., Madara, J.C., Fenselau, H., Goldey, G.J., Diaz, V.E., Jikomes, N., Resch, J.M., et al. (2017). Homeostatic circuits selectively gate food cue responses in insular cortex. Nature.

Mandelblat-Cerf, Y., Ramesh, R.N., Burgess, C.R., Patella, P., Yang, Z., Lowell, B.B., and Andermann, M.L. (2015). Arcuate hypothalamic AgRP and putative POMC neurons show opposite changes in spiking across multiple timescales. eLife 4.

Ng, L., Bernard, A., Lau, C., Overly, C.C., Dong, H.W., Kuan, C., Pathak, S., Sunkin, S.M., Dang, C., Bohland, J.W., et al. (2009). An anatomic gene expression atlas of the adult mouse brain. Nature neuroscience 12, 356-362.

Pauliukonis, A.C., Buenaventura, C., Cline, M.A., and Gilbert, E.R. (2020). Anorexigenic effects of substance $\mathrm{P}$ in Coturnix japonica. Neuropeptides 81, 102045.

Roman, C.W., Derkach, V.A., and Palmiter, R.D. (2016). Genetically and functionally defined NTS to PBN brain circuits mediating anorexia. Nature communications 7, 11905.

Sternson, S.M., and Eiselt, A.K. (2017). Three Pillars for the Neural Control of Appetite. Annual review of physiology 79, 401-423.

Tachibana, T., Khan, M.S., Matsuda, K., Ueda, H., and Cline, M.A. (2010). Central administration of substance P inhibits feeding behavior in chicks. Hormones and behavior 57, 203-208.

Taniguchi, H., He, M., Wu, P., Kim, S., Paik, R., Sugino, K., Kvitsiani, D., Fu, Y., Lu, J., Lin, Y., et al. (2011). A resource of Cre driver lines for genetic targeting of GABAergic neurons in cerebral cortex. Neuron 71, 995-1013.

Wallen-Mackenzie, A., Dumas, S., Papathanou, M., Martis Thiele, M.M., Vlcek, B., Konig, N., and Bjorklund, A.K. (2020). Spatio-molecular domains identified in the mouse subthalamic nucleus and neighboring glutamatergic and GABAergic brain structures. Commun Biol 3, 338.

Wang, D., He, X., Zhao, Z., Feng, Q., Lin, R., Sun, Y., Ding, T., Xu, F., Luo, M., and Zhan, C. (2015). Whole-brain mapping of the direct inputs and axonal projections of POMC and AgRP neurons. Frontiers in neuroanatomy 9, 40.

Wang, Y., Kim, J., Schmit, M.B., Cho, T.S., Fang, C., and Cai, H. (2019). A bed nucleus of stria terminalis microcircuit regulating inflammation-associated modulation of feeding. Nature communications 10, 2769. 
Wu, Q., Boyle, M.P., and Palmiter, R.D. (2009). Loss of GABAergic signaling by AgRP neurons to the parabrachial nucleus leads to starvation. Cell 137, 1225-1234.

Yasoshima, Y., Scott, T.R., and Yamamoto, T. (2006). Memory-dependent c-Fos expression in the nucleus accumbens and extended amygdala following the expression of a conditioned taste aversive in the rat. Neuroscience 141, 35-45.

Zhang, X., and van den Pol, A.N. (2017). Rapid binge-like eating and body weight gain driven by zona incerta GABA neuron activation. Science 356, 853-859.

Zhu, X., Krasnow, S.M., Roth-Carter, Q.R., Levasseur, P.R., Braun, T.P., Grossberg, A.J., and Marks, D.L. (2012). Hypothalamic signaling in anorexia induced by indispensable amino acid deficiency. Am J Physiol Endocrinol Metab 303, E1446-1458.

Zseli, G., Vida, B., Martinez, A., Lechan, R.M., Khan, A.M., and Fekete, C. (2016). Elucidation of the anatomy of a satiety network: Focus on connectivity of the parabrachial nucleus in the adult rat. J Comp Neurol 524, 2803-2827.

Zseli, G., Vida, B., Szilvasy-Szabo, A., Toth, M., Lechan, R.M., and Fekete, C. (2017). Neuronal connections of the central amygdalar nucleus with refeeding-activated brain areas in rats. Brain Struct Funct. 
Figure 1

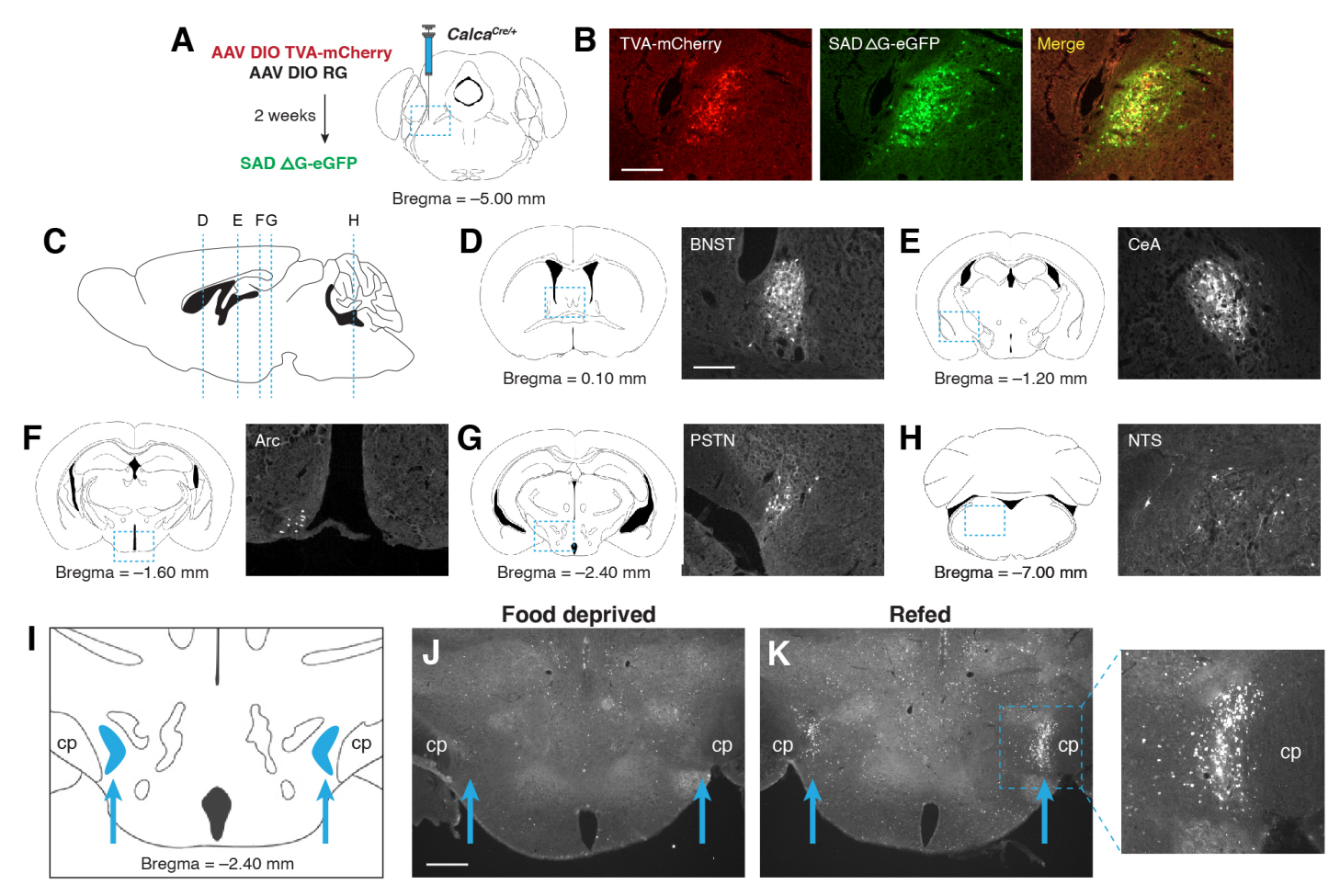




\section{Figure 2}
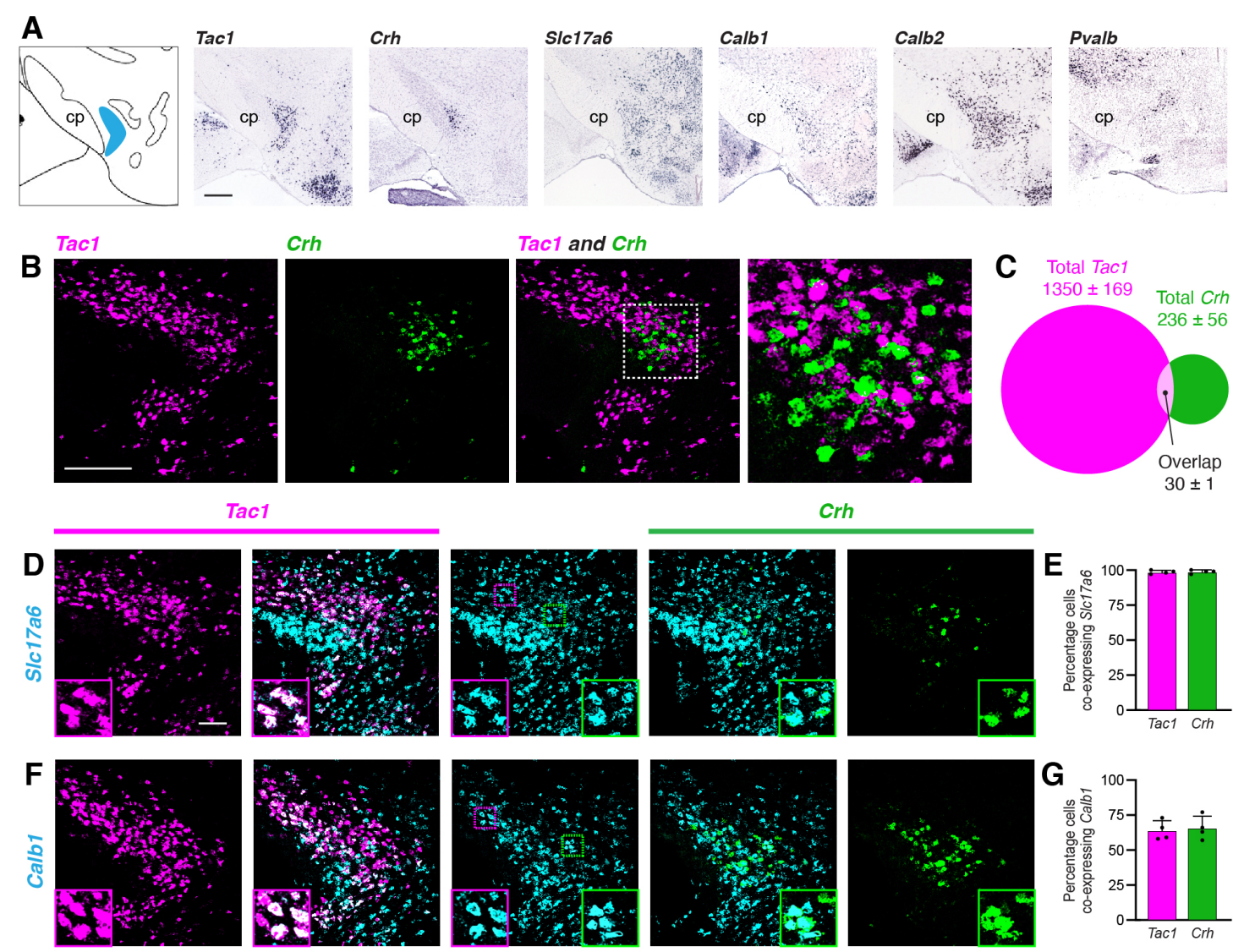

Crh
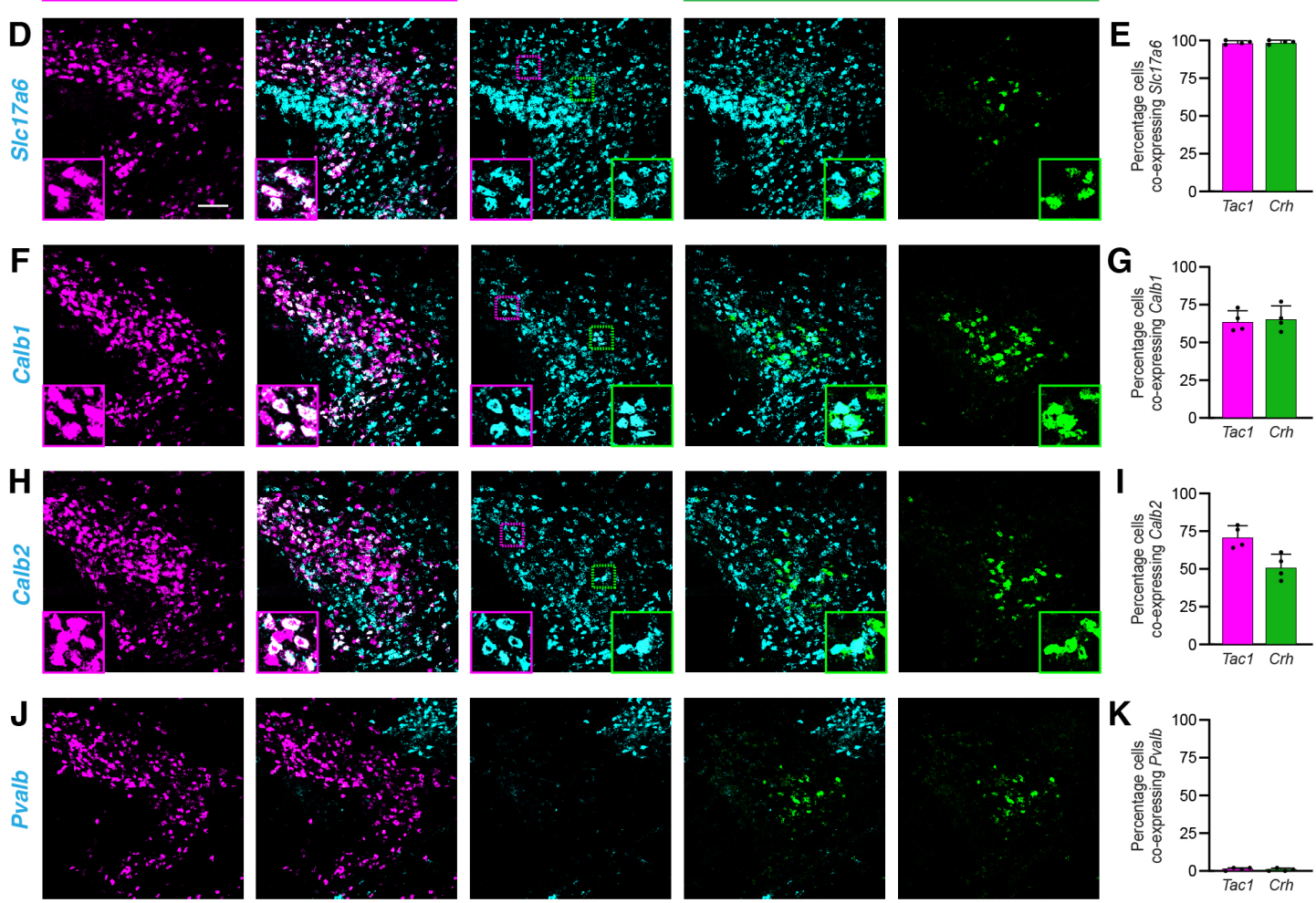


\section{Figure 3}
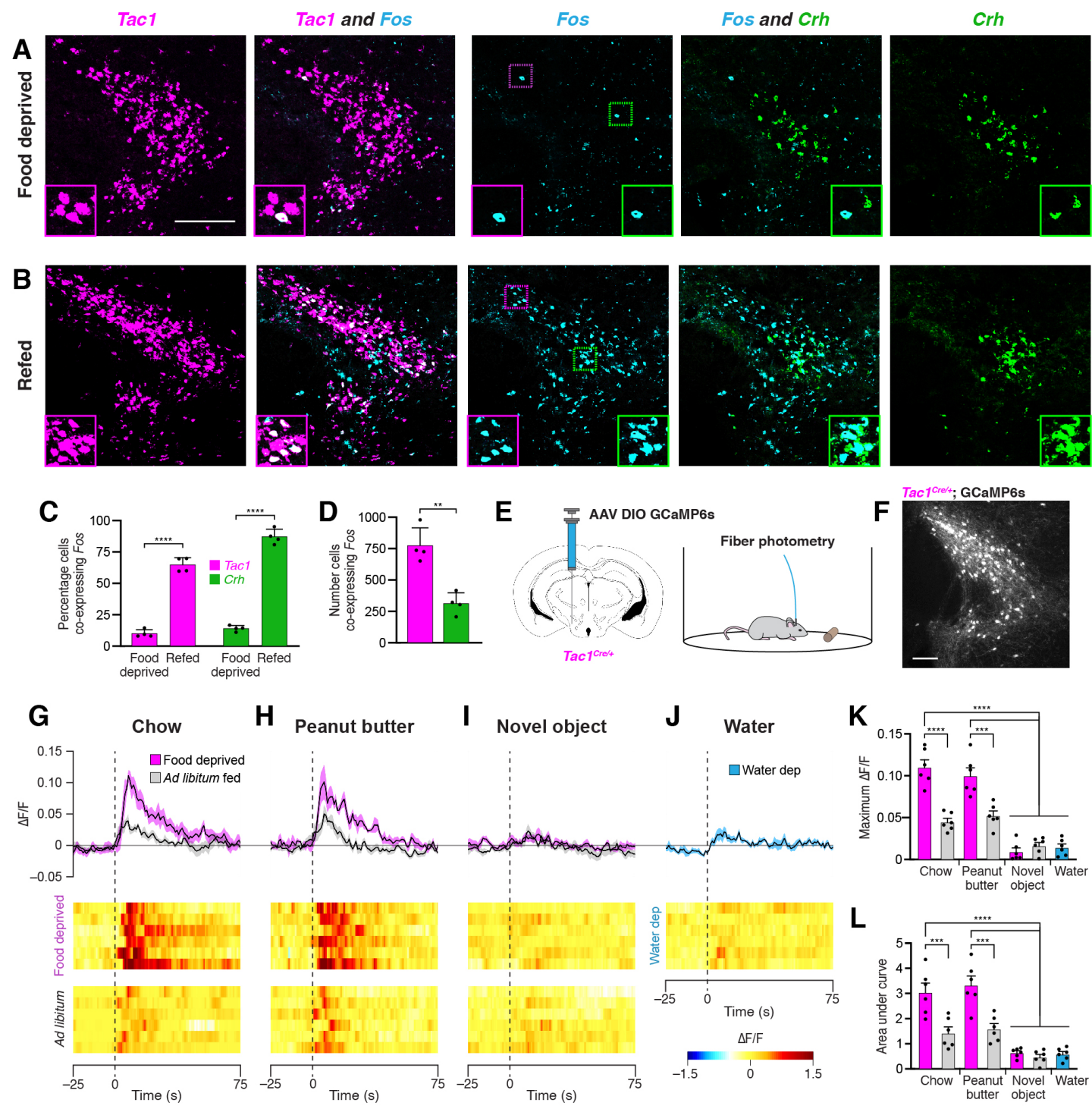

H Peanut butter

I Novel object
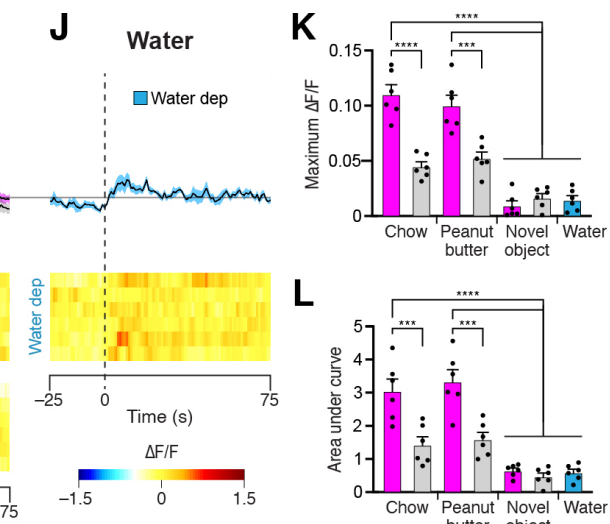

$\mathbf{L}$

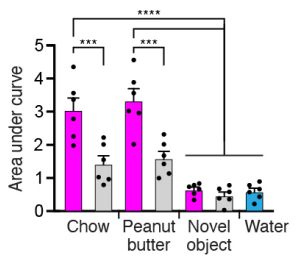




\section{Figure 4}
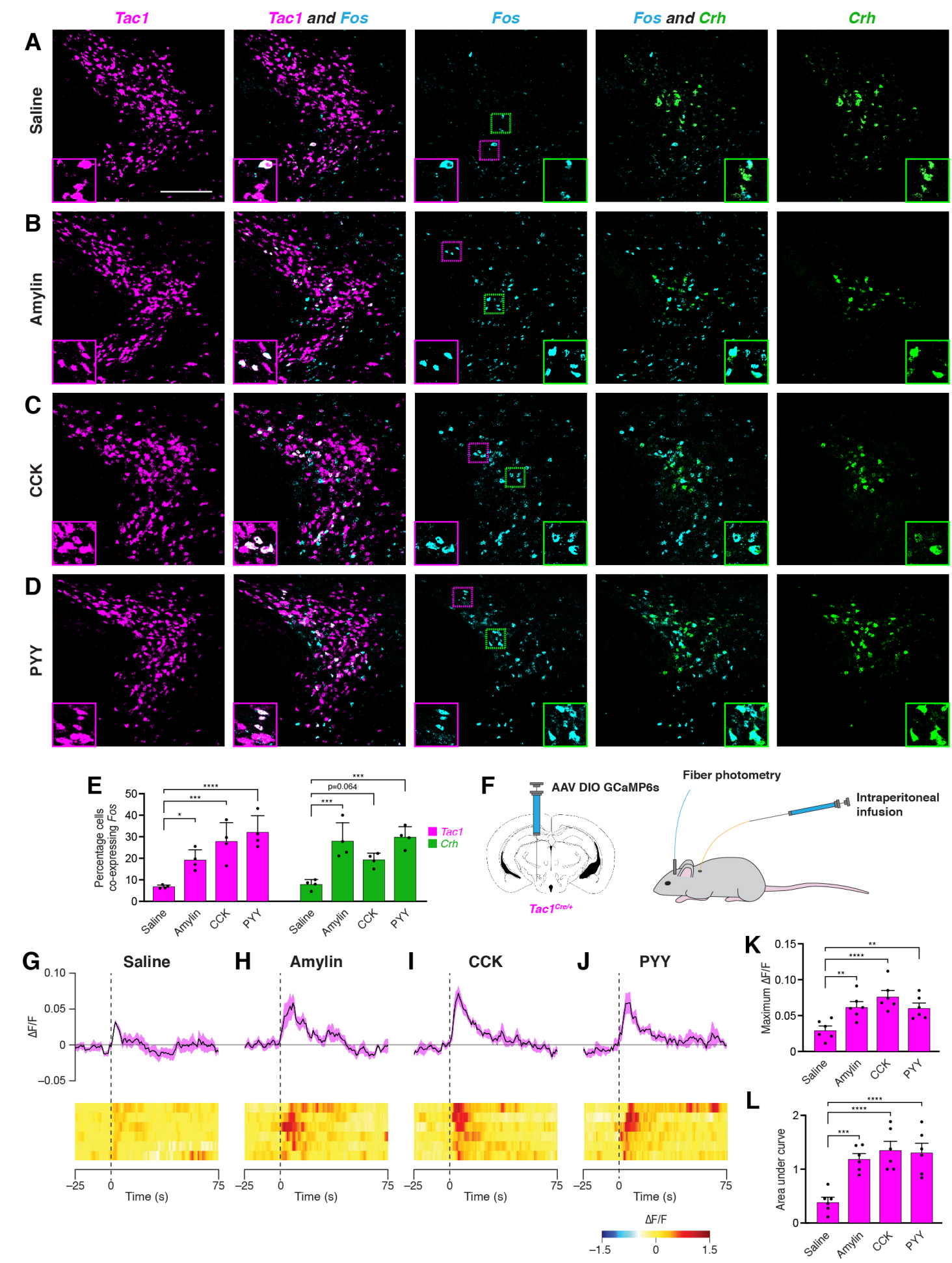

Fiber photometry

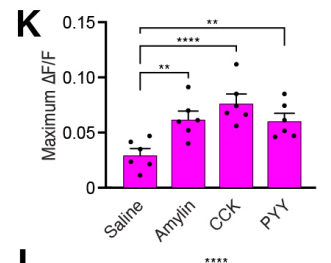

L

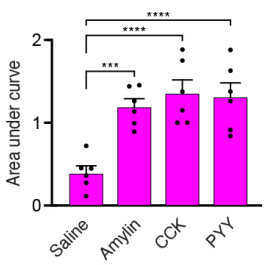


Figure 5

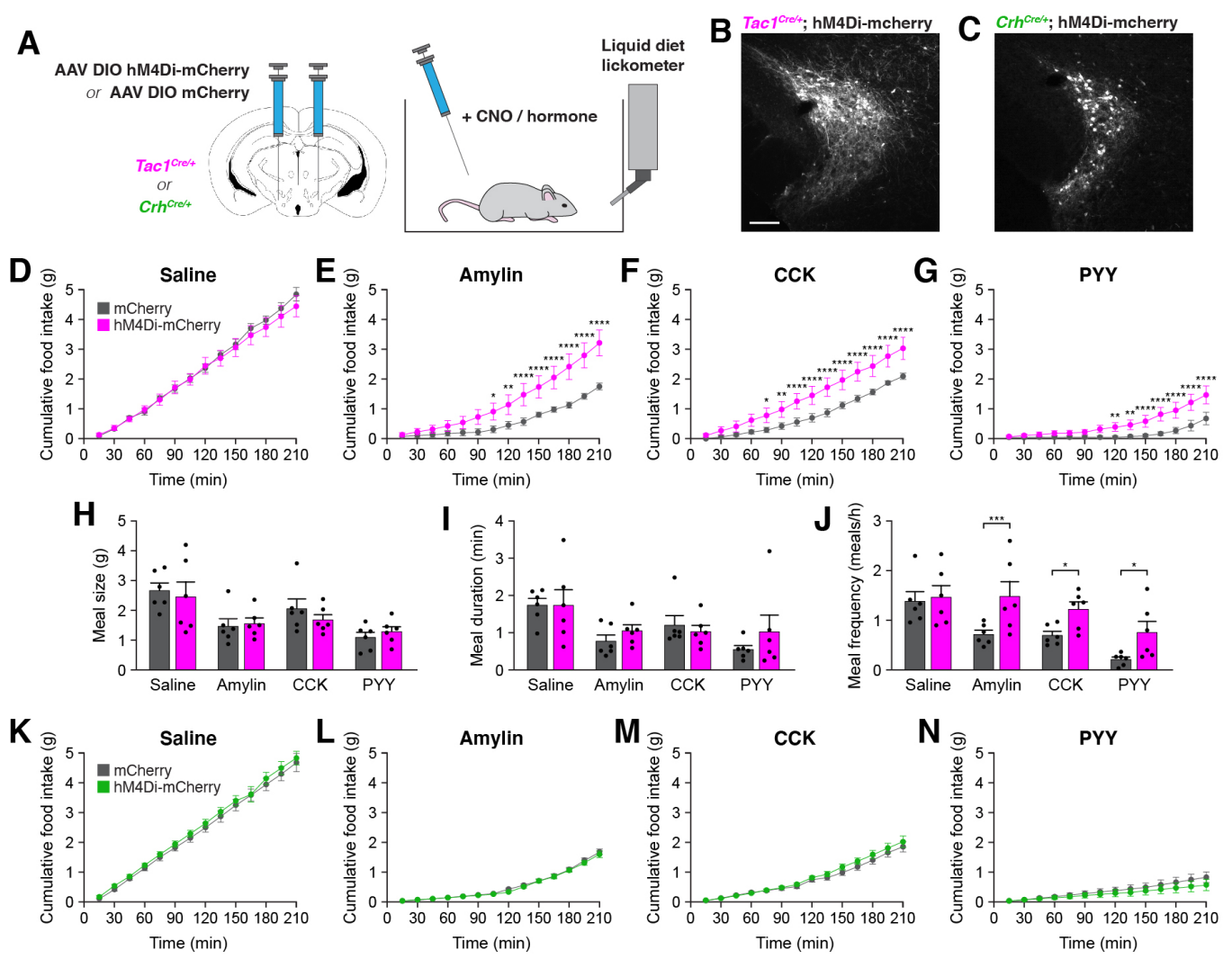




\section{Figure 6}
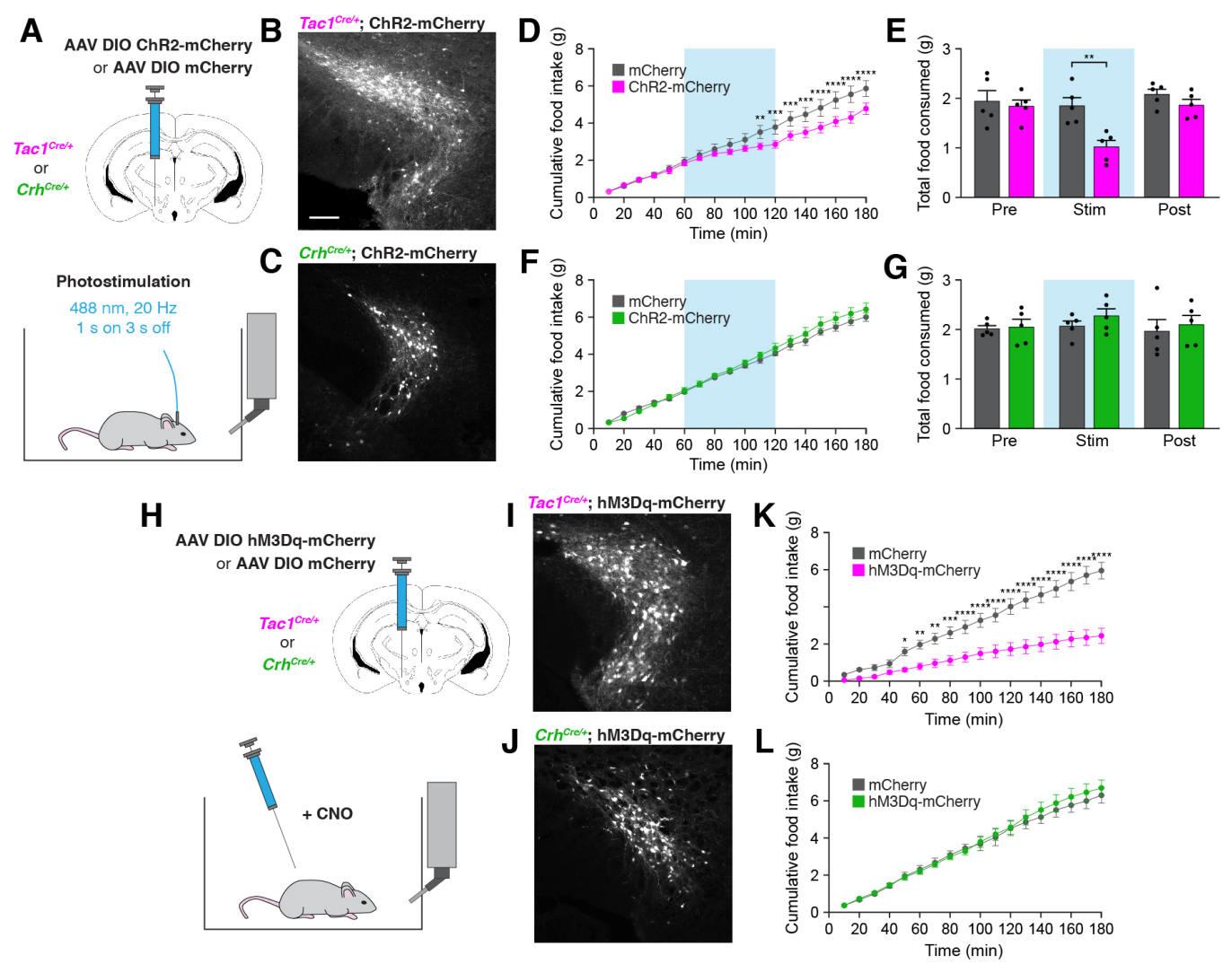


\section{Figure 7}

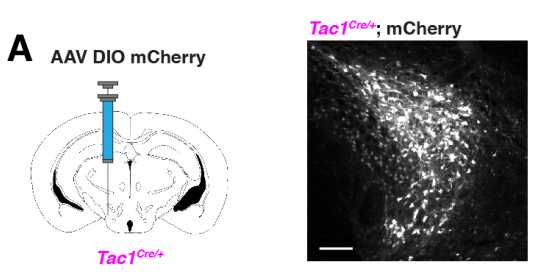

C

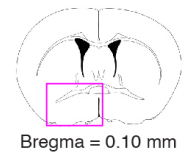

D

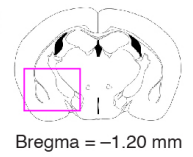

E

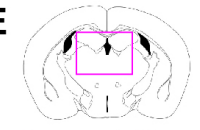

Bregma $=-1.20 \mathrm{~mm}$

$\mathbf{F}$

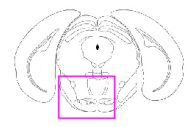

Bregma $=-4.20 \mathrm{~mm}$

G

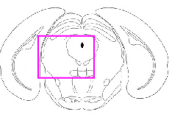

Bregma $=-4.20 \mathrm{~mm}$

H

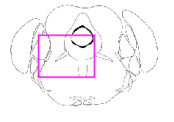

Bregma $=-5.00 \mathrm{~mm}$

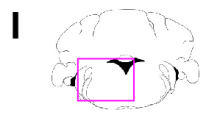

Bregma $=-6.10 \mathrm{~mm}$

J

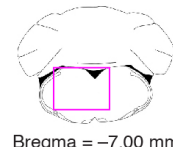

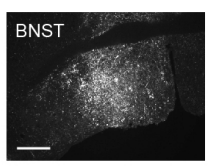
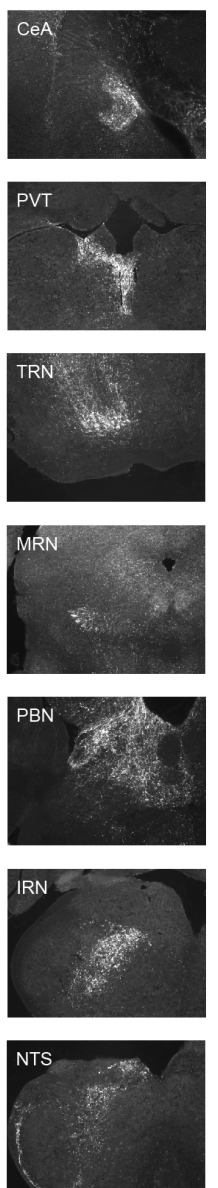
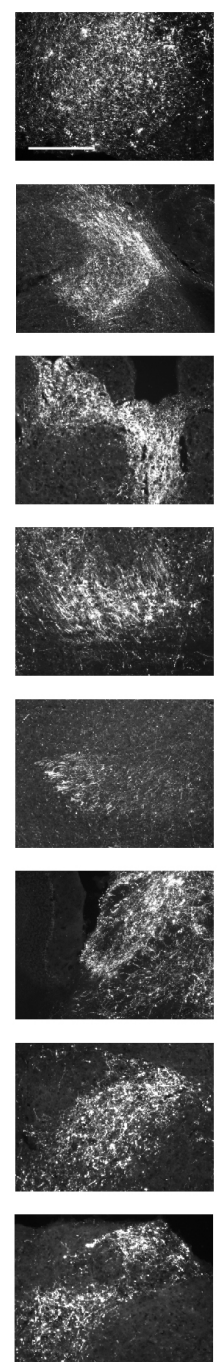

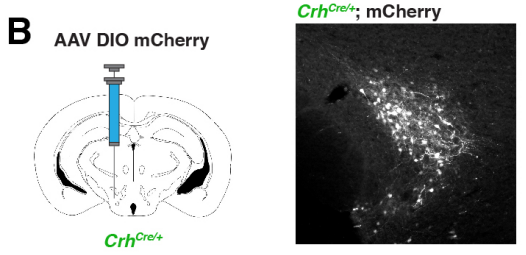

K

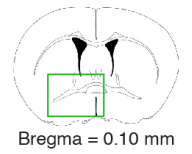

L
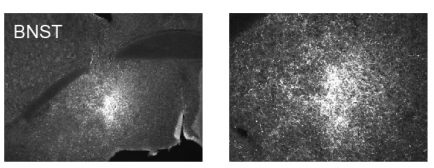

L
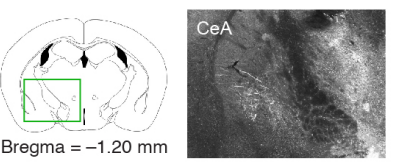

M

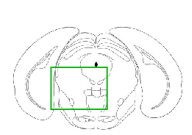

Bregma $=-4.20 \mathrm{~mm}$
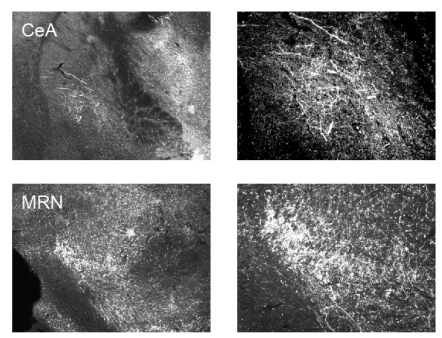

$\mathbf{N}$
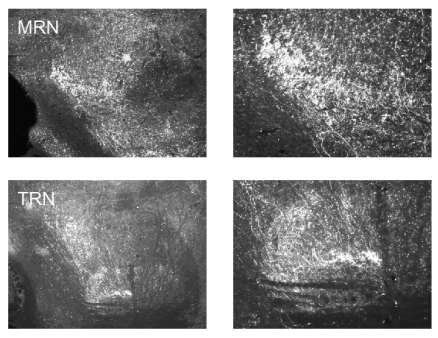

0
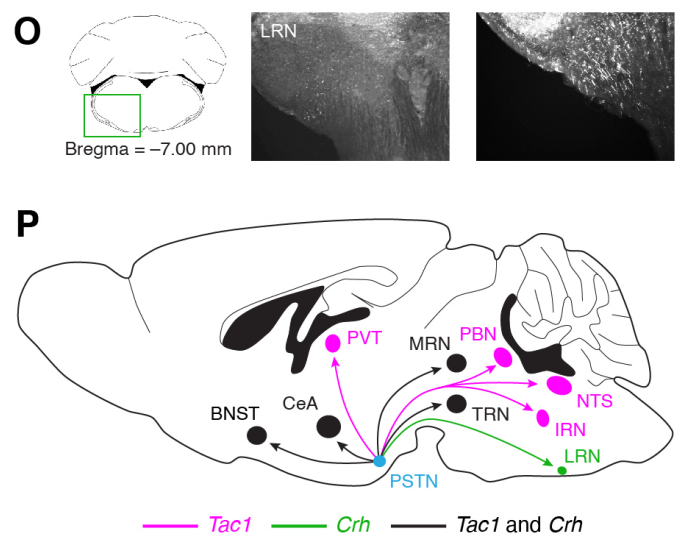


\section{Figure 8}
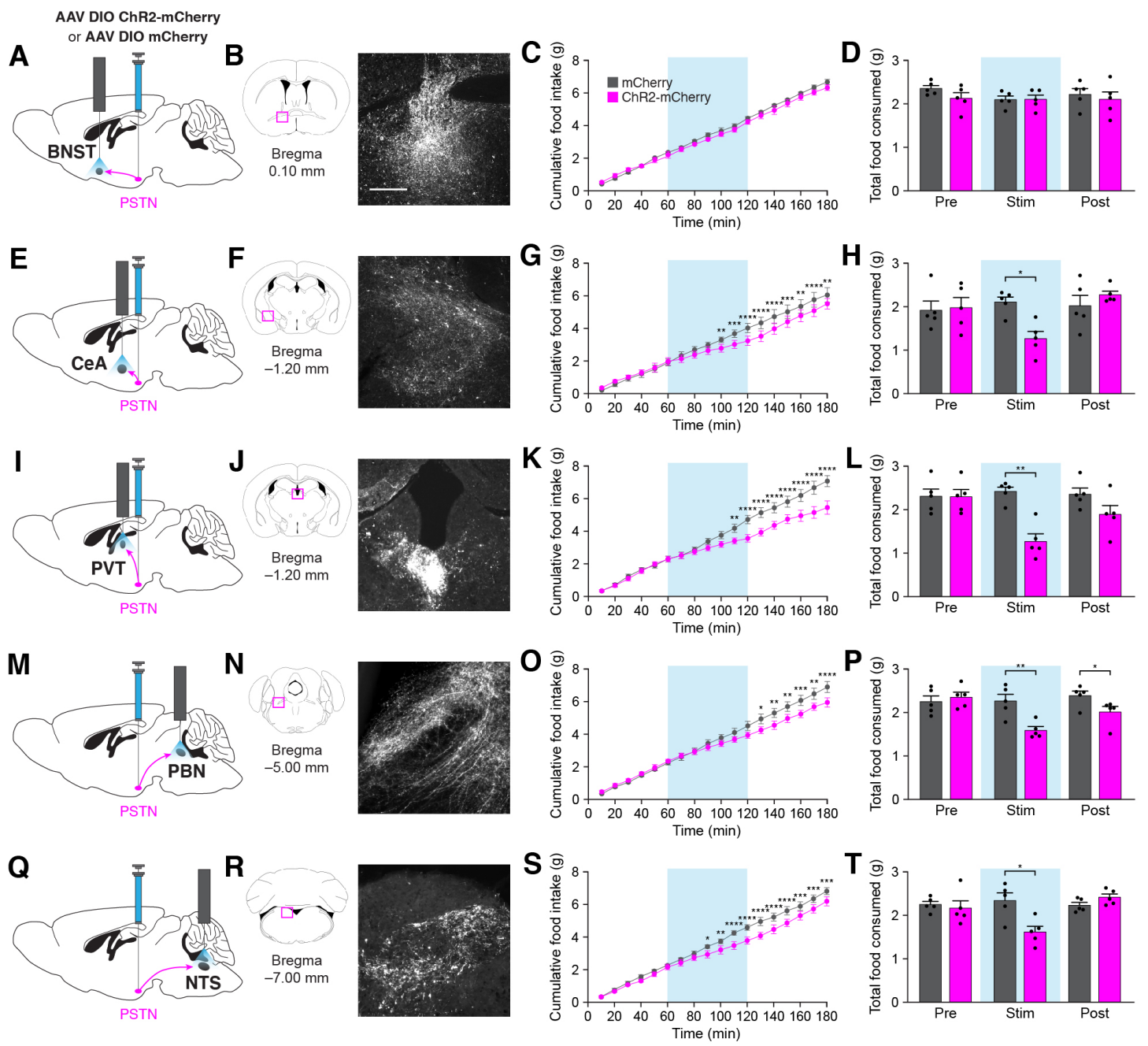


\begin{tabular}{|c|c|c|}
\hline \multicolumn{3}{|c|}{ Supplementary Table 1: Supplementary Statistical Analysis } \\
\hline Figure & Sample Size & Statistical Analysis \\
\hline $3 C$ & $\mathrm{n}=4$ mice & $\begin{array}{l}\text { Two-way ANOVA: significant interaction of cell type } x \text { feeding } \\
\text { conditions: } F_{1,12}=16.11, p=0.0017 \text {. Bonferroni post-hoc analysis shows } \\
\text { significant differences as indicated. }\end{array}$ \\
\hline $3 \mathrm{D}$ & $\mathrm{n}=4$ mice & Student's t-test (unpaired, two-tailed): $\mathrm{t}_{6}=5.533, \mathrm{p}=0.0015$. \\
\hline $3 K$ & $\mathrm{n}=6$ mice & $\begin{array}{l}\text { One-way repeated measures ANOVA: significant effect of stimulus } \\
\text { condition: } F_{6,30}=43.67, p<0.0001 \text {. Bonferroni post-hoc analysis shows } \\
\text { significant differences as indicated. }\end{array}$ \\
\hline $3 \mathrm{~L}$ & $\mathrm{n}=6$ mice & $\begin{array}{l}\text { One-way repeated measures ANOVA: significant effect of stimulus } \\
\text { condition: } F_{6,30}=25.56, p<0.0001 \text {. Bonferroni post-hoc analysis shows } \\
\text { significant differences as indicated. }\end{array}$ \\
\hline $4 \mathrm{E}$ & $\mathrm{n}=4$ mice & $\begin{array}{l}\text { Two-way ANOVA: significant interaction of cell type } x \text { hormones: } F_{3,24}= \\
3.019, p=0.0495 \text {. Bonferroni post-hoc analysis shows significant } \\
\text { differences as indicated. }\end{array}$ \\
\hline $4 K$ & $\mathrm{n}=6$ mice & $\begin{array}{l}\text { One-way repeated measures ANOVA: significant effect of hormone } \\
\text { condition: } F_{3,15}=13.84, p=0.0001 \text {. Bonferroni post-hoc analysis shows } \\
\text { significant differences as indicated. }\end{array}$ \\
\hline $4 \mathrm{~L}$ & $\mathrm{n}=6$ mice & $\begin{array}{l}\text { One-way repeated measures ANOVA: significant effect of hormone } \\
\text { condition: } F_{3,15}=20.66, p<0.0001 \text {. Bonferroni post-hoc analysis shows } \\
\text { significant differences as indicated. }\end{array}$ \\
\hline $5 \mathrm{D}$ & $\mathrm{n}=6$ mice & $\begin{array}{l}\text { Two-way repeated measures ANOVA: no significant interaction of time } \\
x \text { transgene: } F_{14,70}=1.738, p=0.0672 \text {. }\end{array}$ \\
\hline $5 E$ & $\mathrm{n}=6$ mice & $\begin{array}{l}\text { Two-way repeated measures ANOVA: significant interaction of time } x \\
\text { transgene: } F_{14,70}=7.617, p<0.0001 \text {. Bonferroni post-hoc analysis } \\
\text { shows significant differences as indicated. }\end{array}$ \\
\hline $5 F$ & $\mathrm{n}=6$ mice & $\begin{array}{l}\text { Two-way repeated measures ANOVA: significant interaction of time } \mathrm{x} \\
\text { transgene: } \mathrm{F}_{14,70}=5.332, \mathrm{p}<0.0001 \text {. Bonferroni post-hoc analysis shows } \\
\text { significant differences as indicated. }\end{array}$ \\
\hline $5 G$ & $\mathrm{n}=6$ mice & $\begin{array}{l}\text { Two-way repeated measures ANOVA: significant interaction of time } \mathrm{x} \\
\text { transgene: } \mathrm{F}_{14,70}=8.421, \mathrm{p}<0.0001 \text {. Bonferroni post-hoc analysis shows } \\
\text { significant differences as indicated. }\end{array}$ \\
\hline $5 \mathrm{H}$ & $\mathrm{n}=6$ mice & $\begin{array}{l}\text { Two-way repeated measures ANOVA: no significant interaction of } \\
\text { hormone condition } x \text { transgene: } F_{3,15}=0.7227, p=0.5540 \text {. }\end{array}$ \\
\hline 51 & $\mathrm{n}=6$ mice & $\begin{array}{l}\text { Two-way repeated measures ANOVA: no significant interaction of } \\
\text { hormone condition } x \text { transgene: } F_{3,15}=0.5623, p=0.6481 \text {. }\end{array}$ \\
\hline 5J & $\mathrm{n}=6$ mice & $\begin{array}{l}\text { Two-way repeated measures ANOVA: significant interaction of } \\
\text { hormone condition } x \text { transgene: } F_{3,15}=4.670, p=0.0170 \text {. Bonferroni } \\
\text { post-hoc analysis shows significant differences as indicated. }\end{array}$ \\
\hline
\end{tabular}




\begin{tabular}{|c|c|c|}
\hline $5 K$ & $\mathrm{n}=6$ mice & $\begin{array}{l}\text { Two-way repeated measures ANOVA: no significant interaction of time } \\
x \text { transgene: } F_{14,70}=0.1535, p=0.9998 \text {. }\end{array}$ \\
\hline $5 \mathrm{~L}$ & $\mathrm{n}=6$ mice & $\begin{array}{l}\text { Two-way repeated measures ANOVA: no significant interaction of time } \\
x \text { transgene: } F_{14,70}=0.4719, p=0.9407\end{array}$ \\
\hline $5 \mathrm{M}$ & $\mathrm{n}=6$ mice & $\begin{array}{l}\text { Two-way repeated measures ANOVA: no significant interaction of time } \\
x \text { transgene: } F_{14,70}=0.4635, p=0.9448 \text {. }\end{array}$ \\
\hline $5 N$ & $\mathrm{n}=6$ mice & $\begin{array}{l}\text { Two-way repeated measures ANOVA: no significant interaction of time } \\
x \text { transgene: } F_{14,70}=0.5147, p=0.9168 \text {. }\end{array}$ \\
\hline $6 \mathrm{D}$ & $\mathrm{n}=5$ mice & $\begin{array}{l}\text { Two-way repeated measures ANOVA: significant interaction of time } \mathrm{x} \\
\text { transgene: } \mathrm{F}_{18,72}=5.323, \mathrm{p}<0.0001 \text {. Bonferroni post-hoc analysis shows } \\
\text { significant differences as indicated. }\end{array}$ \\
\hline $6 \mathrm{E}$ & $\mathrm{n}=5$ mice & $\begin{array}{l}\text { Two-way repeated measures ANOVA: significant interaction of time } \mathrm{x} \\
\text { transgene: } \mathrm{F}_{2,8}=5.262, \mathrm{p}=0.0348 \text {. Bonferroni post-hoc analysis shows } \\
\text { significant differences as indicated. }\end{array}$ \\
\hline $6 \mathrm{~F}$ & $\mathrm{n}=5$ mice & $\begin{array}{l}\text { Two-way repeated measures ANOVA: no significant interaction of time } \\
x \text { transgene: } F_{18,72}=1.698, p=0.0596 .\end{array}$ \\
\hline $6 \mathrm{G}$ & $\mathrm{n}=5$ mice & $\begin{array}{l}\text { Two-way repeated measures ANOVA: no significant interaction of time } \\
x \text { transgene: } F_{2,8}=0.5324, p=0.6066 \text {. Bonferroni post-hoc analysis } \\
\text { shows significant differences as indicated. }\end{array}$ \\
\hline $6 K$ & $\mathrm{n}=5$ mice & $\begin{array}{l}\text { Two-way repeated measures ANOVA: significant interaction of time } \mathrm{x} \\
\text { transgene: } \mathrm{F}_{18,72}=12.66, \mathrm{p}<0.0001 \text {. Bonferroni post-hoc analysis shows } \\
\text { significant differences as indicated. }\end{array}$ \\
\hline $6 \mathrm{~L}$ & $\mathrm{n}=5$ mice & $\begin{array}{l}\text { Two-way repeated measures ANOVA: no significant interaction of time } \\
x \text { transgene: } F_{18,72}=0.4668, p=0.9641 \text {. }\end{array}$ \\
\hline $8 C$ & $\mathrm{n}=5$ mice & $\begin{array}{l}\text { Two-way repeated measures ANOVA: significant interaction of time } x \\
\text { transgene: } F_{18,72}=5.212, p<0.0001 \text {. Bonferroni post-hoc analysis } \\
\text { shows significant differences as indicated. }\end{array}$ \\
\hline $8 D$ & $\mathrm{n}=5$ mice & $\begin{array}{l}\text { Two-way repeated measures ANOVA: significant interaction of time } x \\
\text { transgene: } F_{2,8}=6.732, p=0.0193 \text {. Bonferroni post-hoc analysis shows } \\
\text { significant differences as indicated. }\end{array}$ \\
\hline $8 \mathrm{G}$ & $\mathrm{n}=5$ mice & $\begin{array}{l}\text { Two-way repeated measures ANOVA: significant interaction of time } x \\
\text { transgene: } F_{18,72}=4.550, p<0.0001 \text {. Bonferroni post-hoc analysis } \\
\text { shows significant differences as indicated. }\end{array}$ \\
\hline $8 \mathrm{H}$ & $\mathrm{n}=5$ mice & $\begin{array}{l}\text { Two-way repeated measures ANOVA: significant interaction of time } \mathrm{x} \\
\text { transgene: } \mathrm{F}_{2,8}=10.22, \mathrm{p}=0.0063 \text {. Bonferroni post-hoc analysis shows } \\
\text { significant differences as indicated. }\end{array}$ \\
\hline $8 K$ & $\mathrm{n}=5$ mice & $\begin{array}{l}\text { Two-way repeated measures ANOVA: significant interaction of time } \mathrm{x} \\
\text { transgene: } \mathrm{F}_{18,72}=10.24, \mathrm{p}<0.0001 \text {. Bonferroni post-hoc analysis } \\
\text { shows significant differences as indicated. }\end{array}$ \\
\hline
\end{tabular}




\begin{tabular}{|c|c|l|}
\hline $8 \mathrm{~L}$ & $\mathrm{n}=5$ mice & $\begin{array}{l}\text { Two-way repeated measures ANOVA: significant interaction of time } \mathrm{x} \\
\text { transgene: } \mathrm{F}_{2,8}=5.803, \mathrm{p}=0.0277 . \text { Bonferroni post-hoc analysis shows } \\
\text { significant differences as indicated. }\end{array}$ \\
\hline 80 & $\mathrm{n}=5$ mice & $\begin{array}{l}\text { Two-way repeated measures ANOVA: significant interaction of time } \mathrm{x} \\
\text { transgene: } \mathrm{F}_{18,72}=7.051, \mathrm{p}<0.0001 . \text { Bonferroni post-hoc analysis } \\
\text { shows significant differences as indicated. }\end{array}$ \\
\hline $8 \mathrm{P}$ & $\mathrm{n}=5$ mice & $\begin{array}{l}\text { Two-way repeated measures ANOVA: significant interaction of time } \mathrm{x} \\
\text { transgene: } \mathrm{F}_{2,8}=6.437, \mathrm{p}<0.0216 . \text { Bonferroni post-hoc analysis shows } \\
\text { significant differences as indicated. }\end{array}$ \\
\hline $8 \mathrm{~S}$ & $\mathrm{n}=5$ mice & $\begin{array}{l}\text { Two-way repeated measures ANOVA: no significant interaction of time } \\
x \text { transgene: } \mathrm{F}_{18,72}=1.567, \mathrm{p}=0.0928 .\end{array}$ \\
\hline $8 \mathrm{~T}$ & $\mathrm{n}=5$ mice & $\begin{array}{l}\text { Two-way repeated measures ANOVA: no significant interaction of time } \\
x \text { transgene: } \mathrm{F}_{2,8}=0.3862, \mathrm{p}=0.6916 .\end{array}$ \\
\hline
\end{tabular}

